\title{
Weak homology of elliptical galaxies
}

\author{
G. Bertin ${ }^{1,2}$, L. Ciotti ${ }^{1,3}$, and M. Del Principe ${ }^{1,4}$ \\ 1 Scuola Normale Superiore, Piazza dei Cavalieri 7, 56126 Pisa, Italy \\ 2 Università degli Studi di Milano, Dipartimento di Fisica, via Celoria 16, 20133 Milano, Italy \\ e-mail: Giuseppe.Bertin@unimi.it \\ 3 Osservatorio Astronomico di Bologna, via Ranzani 1, 40127 Bologna, Italy \\ e-mail: ciotti@bo.astro.it \\ 4 Università degli Studi dell'Aquila, Dipartimento di Fisica, via Vetoio Località Coppito, 67100 L'Aquila, Italy \\ e-mail: milena@terri1.te.astro.it
}

Received 22 November 2001 / Accepted 8 February 2002

\begin{abstract}
Studies of the Fundamental Plane of early-type galaxies, from small to intermediate redshifts, are generally carried out under the guiding principle that the Fundamental Plane reflects the existence of an underlying mass-luminosity relation for such galaxies, in a scenario where galaxies are homologous systems in dynamical equilibrium. In this paper we re-examine the question of whether a systematic non-homology could be partly responsible for the correlations that define the Fundamental Plane. We start by studying a small set of objects characterized by photometric profiles that have been pointed out to deviate significantly from the standard $R^{1 / 4}$ law. For these objects we confirm that a generic $R^{1 / n}$ law, with $n$ a free parameter, can provide superior fits (the best-fit value of $n$ can be lower than 2.5 or higher than 10), better than those that can be obtained by a pure $R^{1 / 4}$ law, by an $R^{1 / 4}+$ exponential model, and by other dynamically justified self-consistent models. Therefore, strictly speaking, elliptical galaxies should not be considered homologous dynamical systems. Still, a case for weak homology, useful for the interpretation of the Fundamental Plane, could be made if the best-fit parameter $n$, as often reported, correlates with galaxy luminosity $L$, provided the underlying dynamical structure also follows a systematic trend with luminosity. We demonstrate that this statement may be true even in the presence of significant scatter in the correlation $n(L)$. Preliminary indications provided by a set of "data points" associated with a sample of 14 galaxies suggest that neither the strict homology nor the constant stellar massto-light solution are a satisfactory explanation of the observed Fundamental Plane. These conclusions await further extensions and clarifications, because the class of low-luminosity early-type galaxies, which contribute significantly to the Fundamental Plane, falls outside the simple dynamical framework considered here and because dynamical considerations should be supplemented with other important constraints derived from the evolution of stellar populations.
\end{abstract}

Key words. galaxies: elliptical and lenticular, $\mathrm{cD}$ - galaxies: fundamental parameters - galaxies: kinematics and dynamics - galaxies: photometry

\section{Introduction}

In the three-dimensional space of observed parameters $\log \sigma_{0}, \log R_{\mathrm{e}}$, and $S B_{\mathrm{e}}=-2.5 \log \langle I\rangle_{\mathrm{e}} \quad\left(\right.$ where $\langle I\rangle_{\mathrm{e}}=$ $\left.L / 2 \pi R_{\mathrm{e}}^{2}\right)$, early-type galaxies are approximately located on a plane, called the Fundamental Plane (hereafter FP; Dressler et al. 1987; Djorgovski \& Davis 1987; Bender et al. 1992), represented by the best-fit relation

$\log R_{\mathrm{e}}=\alpha \log \sigma_{0}+\beta S B_{\mathrm{e}}+\gamma$.

The central velocity dispersion $\sigma_{0}$ is often defined as the velocity dispersion referred to an aperture radius of $R_{\mathrm{e}} / 8$

Send offprint requests to: M. Del Principe,

e-mail: milena@terri.te.astro.it
(Jørgensen et al. 1993); the quantities $R_{\mathrm{e}}$ and $L$ are the empirically determined half-luminosity (effective) radius and total absolute luminosity. The coefficients $\alpha, \beta$, and $\gamma$ depend slightly on the photometric band considered. By measuring $R_{\mathrm{e}}$ in kpc, $\sigma_{0}$ in $\mathrm{km} \mathrm{s}^{-1}$, and $S B_{\mathrm{e}}=42.0521-$ $2.5 \log \left(L / 2 \pi R_{\mathrm{e}}^{2}\right)$ in $\mathrm{mag} / \operatorname{arcsec}^{2}$, where $L$ is expressed in units of the solar Blue luminosity, reported values are $\alpha=$ $1.25 \pm 0.1, \beta=0.32 \pm 0.03, \gamma=-8.895$ (Jørgensen et al. 1993; Bender et al. 1998; note that these coefficients, in particular this choice of $\gamma$, refer to a long cosmological distance scale). The FP is characterized by a small scatter in $R_{\mathrm{e}}$, on the order of $15 \%$, remarkably independent of the position on the FP itself. 
Studies of the FP of early-type galaxies, from low to intermediate redshifts (for this latter case, see van Dokkum \& Franx 1996; Kelson et al. 1997; van Dokkum et al. 1998a,b; Bender et al. 1998; Pahre et al. 1998a; Pahre et al. 1998b; Jørgensen et al. 1999; Treu et al. 1999; Kelson et al. 2000a,b,c; Treu et al. 2001a,b), are generally carried out under the guiding principle that the FP reflects the existence of an underlying mass-luminosity relation for such galaxies (see Faber et al. 1987; van Albada et al. 1995), in a scenario where galaxies are homologous systems in dynamical equilibrium. According to this simplified picture, galaxies are thought to be characterized by a universal internal structure (density and pressure tensor distributions), which differs from galaxy to galaxy only in terms of the relevant scales. An empirical indication that this may be the case is suggested by the fact that, on the large scale, the luminosity profile of elliptical galaxies appears to be universal.

The empirical $R^{1 / 4}$ luminosity "law" (de Vaucouleurs 1948) has long been recognized to fit the surface brightness profiles of elliptical galaxies successfully, to the point that, in the absence of other (spectroscopic) indicators, elliptical galaxies are routinely identified by means of this characteristic photometric signature. In this sense, it might be argued that on the large scale elliptical galaxies are characterized by a universal luminosity profile and thus they are also likely to be dynamically homologous systems. In practice, if we exclude the central regions of the galaxy, generally affected by seeing or instrumental PSF and sometimes studied separately in view of the possible presence of a massive black hole, the overall $R^{1 / 4}$ fit is characterized by residuals typically of the order of $0.1-0.2 \mathrm{mag} / \operatorname{arcsec}^{2}$ (e.g., see de Vaucouleurs \& Capaccioli 1979; Capaccioli 1987; de Carvalho \& da Costa 1988; Capaccioli 1989; Burkert 1993). These deviations from the $R^{1 / 4}$ law, although small, are often larger than the typical observational errors involved. Interestingly, they are systematic and appear to correlate with the galaxy luminosity (Michard 1985; Schombert 1986; see additional comments in the following paragraph). In general, the surface brightness profile in the external regions is found to be flatter for more luminous galaxies.

A simple and popular way to model the systematic non-homology noted in the surface brightness distribution of early-type galaxies is to consider an $R^{1 / n}$ luminosity profile, with $n$ a free parameter, as a generalization of the $R^{1 / 4}$ law (Sersic 1968; see also Davies et al. 1988; Capaccioli 1989; Caon et al. 1993, hereafter CCD93; Young \& Currie 1994; D’Onofrio et al. 1994, hereafter DCC94; Prugniel \& Simien 1997, hereafter PS97; Wadadekar et al. 1999). The $R^{1 / n}$ "law" has been found to be a statistically convenient description to address some issues related to the FP of elliptical galaxies (see Graham et al. 1996; Ciotti et al. 1996; Graham \& Colless 1997; Ciotti \& Lanzoni 1997; Graham 1998). In addition, it has been applied to the description of the surface brightness profiles of galaxy bulges (see Andredakis et al. 1995; Courteau et al. 1996). On the theoretical side, it has been the focus of several general investigations (see, e.g., Makino et al. 1990; Ciotti 1991; Gerbal et al. 1997; Andredakis 1998; Ciotti \& Bertin 1999). If applicable, this description would bridge the gap between the classical $R^{1 / 4}$ profile and the exponential profile characteristic of disks. It has soon become clear that the shape parameter $n$ of the $R^{1 / n}$ law correlates with global quantities such as total luminosity and effective radius (CCD93; PS97; see also Khosroshahi et al. 2000). For example, on a sample of $52 \mathrm{E} / \mathrm{S} 0$ galaxies with $M_{\mathrm{B}}<-17.3$, CCD93 find the following relation:

$\log n=0.28+0.52 \log R_{\mathrm{e}}$

with $\mathrm{rms}=0.18$. Here $n$ is the best-fit value obtained by fitting the luminosity profile along the major axis, and $R_{\mathrm{e}}$ is determined from the curves of growth, independently of the fit procedure. CCD93 noted that the transition from normal to bright ellipticals occurs at $n=4, R_{\mathrm{e}}=3 \mathrm{kpc}$, while low-luminosity ellipticals (Davies et al. 1983) are characterized by $n \approx 1$. The above relation implies the existence of a similar relation between $n$ and $M_{\mathrm{B}}$, since $R_{\mathrm{e}}$ and $M_{\mathrm{B}}$ correlate with each other. For example, CCD93 and DCC94 found:

$n=-19.082-1.211 M_{\mathrm{B}} \simeq-19.082+3.0275 \log L$.

The average scatter around this relation turns out to be $\langle\Delta n\rangle \sim 1.895$, but the effective scatter increases significantly with $L$.

Some confusion has accompanied the application of the $R^{1 / n}$ models to observed objects. For example, PS97 report the correlations $n-R_{\mathrm{e}}$ and $n-M_{\mathrm{B}}$ for a broad sample of ellipticals; but here, in contrast with CCD93, the correlations are shown to saturate, for $\log R_{\mathrm{e}}[\mathrm{kpc}]>0.8$ and $M_{\mathrm{B}}<-20$, to values of $n$ close to 4 . The apparent discrepancy is to be traced to the fitting procedure used. In fact, PS97 referred to the curves of growth (see comments in Appendix B.1 below). As a result, for a given sample of early-type galaxies, values of $n$ not larger than 6 have been claimed by PS97, based on the use of curves of growth, while values of $n$ up to 15 have been found (see CCD93; DCC94) based on direct profile fitting. Curiously, for the low-luminosity objects, where the latter procedure sets values of $n$ below 5 , the two methods appear to be mutually consistent.

Another natural way to address systematic deviations from the $R^{1 / 4}$ law is to imagine (e.g., see Saglia et al. 1997) that the galaxy results from the superposition of an $R^{1 / 4}$ component (presumably spheroidal) and an exponential component (presumably flat). This latter procedure is appealing from the dynamical point of view (S0s are often thought to be made like this), yet the procedure is usually applied as a purely photometric method (with no check on its dynamical foundation; attempts in this latter direction have been made, see Scorza \& Bender 1995).

In view of constructing a physical rationale at the basis of the FP, the existence of strict homology is not necessary (see Ciotti \& Pellegrini 1992; CCD93; 
Renzini \& Ciotti 1993; Djorgovski 1995; Capelato et al. 1985; Hjorth \& Madsen 1995; Ciotti et al. 1996; PS97; Pahre \& Djorgovski 1997; and references therein). In fact, many of the arguments that allow for a physical interpretation of the observed scaling law would carry through even in the case that we may call of weak homology. This is a condition by which the structure and dynamics (density and pressure tensor distributions) of early-type galaxies vary systematically with galaxy luminosity. An indication that this may be a realistic picture is indeed provided by the systematic trends noted above in terms of $R^{1 / n}$ fits. However, we should emphasize that, based on the photometric properties alone of early-type galaxies, we cannot draw conclusions about the FP. For this latter goal we have to make use of dynamical models, because we need to translate the trends observed in the photometric profiles into trends of the relevant coefficients entering the virial theorem. The models used must at least be generally consistent with the available kinematical properties of early-type galaxies, otherwise the consequences drawn would be based on unjustified models. An interesting attempt at combining photometric and kinematic information towards a comprehensive interpretation of the FP has been made recently by Gerhard et al. (2001). However, their detailed dynamical investigation of a sample of 21 galaxies is far from being conclusive, because their sample is mostly biased in the direction of luminous objects and because their kinematical measurements, although based on the sophisticated technique of fitting line profiles, are admittedly subject to significant model limitations and large uncertainties.

We should emphasize that observations already provide important empirical evidence both against strict homology and against the constant stellar mass-to-light ratio hypothesis. On the one hand, the existence of a relation such as that given in Eq. (3) and the fact that trends in the dark matter content with luminosity are noted readily argue against strict homology. On the other hand, the existence of clear correlations involving the galaxy luminosity, such as the color-luminosity relation, and the fact that the coefficients that define the FP depend on the observed waveband suggest that systematic changes in the underlying stellar populations are involved. At the interpretation level, an explanation based on a pure age-luminosity relation is not viable, because it is hard to reconcile with the small and approximately uniform scatter of the FP at zero redshift and because it turns out to be incompatible with the observed properties of the FP at intermediate redshifts. In addition, it has been shown that interpretations based solely on either a dark matter-luminosity relation or a metallicity-luminosity relation or a systematic change of the Initial Mass Function with luminosity are not satisfactory because they do not survive a close scrutiny. In this paper we will add further internal consistency to the arguments in favor of a form of weak homology at the basis of the FP.

In Sect. 2 we carry out an in-depth analysis of 4 significant cases of deviations from the $R^{1 / 4}$ law by analyzing the surface brightness profiles of NGC 1379, NGC 4374, NGC 4458, and NGC 4552. We will confirm that a generic $R^{1 / n}$ law, with $n$ a free parameter, can provide superior fits (the best-fit value of $n$ can be lower than 2.5 or higher than 10), better than those that can be obtained by a pure $R^{1 / 4}$ law, by an $R^{1 / 4}+$ exponential model, and by other dynamically justified self-consistent models. Although it may be argued that four galaxies are a sample of little statistical significance, the detailed tests that we have performed on these otherwise normal objects and, especially, the systematic trends with luminosity noted in the literature for large samples (see Eq. (3), based on a sample of 52 galaxies) provide convincing evidence that homology cannot be considered a strict rule for early-type galaxies.

In Sect. 3 we focus on the main implications of these conclusions on the problem of the tilt and the thickness of the FP. For a constant (from galaxy to galaxy) stellar mass-to-light ratio a suitable correlation $n(L)$ could be consistent with the observed FP. We demonstrate that this statement may be true even in the presence of significant scatter in the correlation $n(L)$, by means of a general mapping procedure from the space of the intrinsic physical model quantities to the space of observed quantities. We also show that, in principle, in the absence of strong empirical constraints on the correlation $n(L)$, the Fundamental Plane could be realized in an infinite number of ways, two of which are indeed the constant stellar mass-to-light solution, and the strict $R^{1 / 4}$ homology solution combined with a suitable mass-luminosity relation. Actually, indications provided by a set of "data points" associated with a sample of 14 galaxies are shown to suggest that neither the strict homology nor the constant stellar mass-to-light solution are a satisfactory explanation of the observed Fundamental Plane.

In the concluding Sect. 4, we briefly address some interesting consequences on the expected trends of the observed FP at intermediate redshifts and outline some natural possible extensions of the work presented here.

Background work is recorded in the Appendices. Appendix A summarizes the properties of the models used in the fits presented in Sect. 2. Appendix B describes the relevant fitting procedures. Appendix $\mathrm{C}$ illustrates some properties of isotropic dynamical models in relation to the definition of the central velocity dispersion $\sigma_{0}$. Finally, Appendix D records the calculation of the virial coefficient associated with the dynamical models considered in this paper.

\section{Deviations from the $R^{1 / 4}$ law}

\subsection{Preliminaries}

Many studies have already addressed the issue of the universality of the $R^{1 / 4}$ law (e.g., see Burkert 1993; CCD93; DCC94; PS97; and references therein). In this paper we would like to focus on some relatively round objects, for which a significant departure from the $R^{1 / 4}$ law has been noted. These objects will be used as benchmark prototypes on which detailed tests will be performed about the 
quality of a number of photometric models, about various effects associated with the adopted fitting procedure, and about the role of the radial range over which the photometric fit is made.

In general, photometric profiles are compared with the $R^{1 / 4}$ law, or with other photometric models, only over a finite radial interval $\left(R_{\min }, R_{\max }\right)$. The inner limit is often taken so as to avoid a detailed discussion of the effects of seeing or instrumental PSF (the effects of the PSF on the observed surface brightness profile become negligible beyond a radial distance from the galaxy center of $2-3$ times the scale of the relevant FWHM; e.g., see Bertin et al. 1988); furthermore, the innermost region of a galaxy may present features that are related to the presence of a massive central black hole, which is generally studied as a separate issue. The outer limit often reflects the quality of the available data, since special care has to be taken to properly subtract the contribution of the sky surface brightness (special techniques make it possible to measure reliable photometric profiles down to $\mu_{\mathrm{B}} \sim 28-29 \mathrm{mag} / \operatorname{arcsec}^{2}$ ). Of course, a correct estimate of the sky background is considerably more difficult in the case of galaxies with nearby companions.

Over a rather small radial range, a very good fit can be obtained rather easily and it is thus hard to discriminate among different models. For example, within the radial range $0.1 R_{\mathrm{e}} \leq R \leq 1.5 R_{\mathrm{e}}$, the galaxies NGC 1379 and NGC 4374 are well fitted by the $R^{1 / 4}$ law. In fact, based on the definition of average residual

$\langle\Delta \mu\rangle=\frac{1}{\sqrt{N}}\left\{\sum_{i=1}^{N}\left[\mu_{1 / 4}\left(R_{i}\right)-\mu_{\mathrm{obs}}\left(R_{i}\right)\right]^{2}\right\}^{1 / 2}$,

where $N$ is the number of photometric data points $\mu_{\mathrm{obs}}$ inside the radial range of the fit, and of maximum residual

$$
(\Delta \mu)_{\max }=\max _{R_{\min } \leq R_{i} \leq R_{\max }}\left|\mu_{1 / 4}\left(R_{i}\right)-\mu_{\mathrm{obs}}\left(R_{i}\right)\right|,
$$

the galaxies NGC 1379 and NGC 4374 are found to be characterized by $\langle\Delta \mu\rangle=0.033 \mathrm{mag} / \operatorname{arcsec}^{2},(\Delta \mu)_{\max }=$ $0.079 \mathrm{mag} / \operatorname{arcsec}^{2}$ and by $\langle\Delta \mu\rangle=0.052 \mathrm{mag} / \operatorname{arcsec}^{2}$, $(\Delta \mu)_{\max }=0.108 \mathrm{mag} /$ arcsec $^{2}$ respectively (Burkert 1993). As we will see, these results change significantly when the radial range of the fit is increased (but see Makino et al. 1990).

\subsection{The sample}

In the following we consider four apparently round "prototypical objects", NGC 1379, NGC 4374, NGC 4458, and NGC 4552, that are known to present significant deviations from the standard $R^{1 / 4}$ luminosity distribution. We refer to photometric data (in the Blue band) available in the literature, taken from Caon et al. (1990) and Caon et al. (1994), that have pointed out the existence of a departure from the $R^{1 / 4}$ behavior. Indeed, we have selected the four galaxies, from the sample of 80 galaxies studied by these authors, based on the following criteria: (1) round isophotes; (2) radially extended available photometry; (3) significant deviations from the $R^{1 / 4}$ law. At this stage, we are not interested in the small-scale behavior of the luminosity distribution at very small galactocentric radii and so we did not look up high-resolution data from more modern investigations (e.g., from HST; see Stiavelli et al. 2001), which would address issues beyond the goals of this paper. On purpose, the adopted criteria 1)-3) do not refer to any, possibly available, kinematical information on the four objects, because we whish to conform to the usual (i.e., purely photometric) selection rules adopted in the construction of early-type galaxy samples for studies of the FP.

For this set of objects we consider the relative performance of four different photometric models: (i) $R^{1 / n}$ (one free dimensionless parameter: $n$ ), (ii) $R^{1 / 4}$ (no free parameters), (iii) $R^{1 / 4}+$ exponential (two free parameters: $L_{\exp } / L_{\text {tot }}$ and $R_{\exp } / R_{1 / 4}$ ), and (iv) $f_{\infty}$ models (one free parameter: $\Psi)$. The main properties of the models are summarized in Appendix A. In all cases, in addition to the best-fit values of the free dimensionless parameters, if available, the fitting procedure leads to the determination of two scales, i.e., of the model effective radius $R_{\mathrm{e}}$ and of the model total luminosity $L$.

Each of the four different photometric models is tested against a variety of options for the fitting procedure: (a) profile fitting or circularized profile fitting or curve of growth fitting, (b) reduced or extended radial range, and (c) constrained or unconstrained integrated luminosity. These fitting procedures are briefly described in Appendix B.

\subsection{Relative performance of four photometric models for four prototypical objects}

In Table 1 we record the results for the best-fit $R^{1 / n}$ models, for the reference option of using circularized profiles, of considering the most extended radial range available, and of imposing no constraint on the integrated luminosity. The quality of the fit is measured by the parameter $\chi^{2}$, defined in Appendix B, and is also described here by the values of $\langle\Delta \mu\rangle$ and $(\Delta \mu)_{\max }$ (see Eqs. (4)-(5)). Some of the digits of the various entries are not significant, but we have decided to keep them since there should be no reason for misinterpretation. The last two columns record the surface brightness excursion available for the photometric data and the total absolute magnitude of each object. The best-fit $R^{1 / n}$ models are then illustrated in Figs. 1 and 2 .

Similarly, in Table 2 we summarize the corresponding performance of the other three photometric models (for the same option of fitting procedure as in Table 1). It is clear that, especially for the two objects (NGC 4374 and NGC 4552) that are characterized by high values of $n$, the model effective radii thus determined by the fit can vary dramatically (but see comments in Sect. 2.4). The fits are then illustrated in Figs. 3-5. For the low$n$ galaxy NGC 1379 , the $f_{\infty}$ models are not providing a 

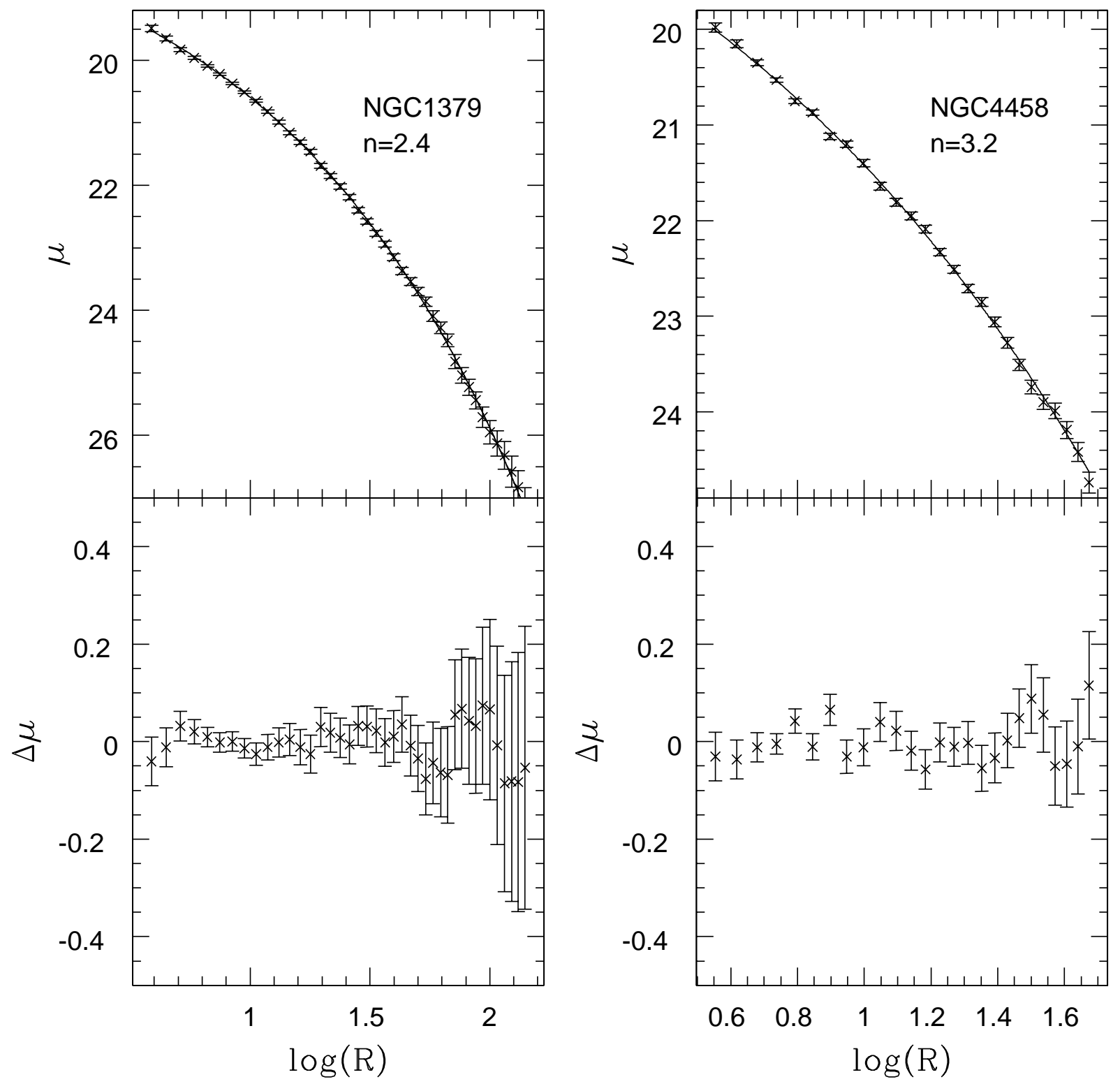

Fig. 1. $R^{1 / n}$ fit. $R$ is in arcsec, $\mu$ in mag/arcsec ${ }^{2}$. The data points in Figs. $1-5$ are in the Blue band, taken from Caon et al. (1990) and Caon et al. (1994). Note the limiting surface brightness reached for each galaxy.

satisfactory fit, which is not surprising since for this galaxy the role of rotation is likely to be important (see the kinematical profile recorded by Graham et al. 1998) and thus the galaxy falls outside the physical scenario at the basis of the models. In addition, two minima in $\chi^{2}$ are actually available for the $f_{\infty}$ models for this galaxy: the higher $\chi^{2}$ model (lower $\Psi$; not illustrated in Fig. 5) is less bright than the galaxy in the central region (opposite to the behavior of the model fit shown in the figure), both models are brighter than the galaxy at large radii.

In Table 3 we summarize the results of the best-fit $R^{1 / n}$ models obtained under various fitting options. It is clear that many of the quantities thus derived are sensitive to the adopted fitting procedure. As an extreme example, we point to the case of NGC 4374, for which the bestfit model for the reduced radial range (case $b$ of Table 3 ) would be characterized by $R_{\mathrm{e}}=1092$ arcsec, so that most of the total luminosity of the galaxy would be assigned to the unobserved part of the photometric profile. Our results quantify and generally confirm trends already noted in earlier studies (see Introduction; but see also some important remarks in Appendix B.1).

For the set of four galaxies, considered to be as prototypes of objects with a genuine deviation from the $R^{1 / 4}$ law, we have found the following results. These are based on direct inspection of Figs. 1-5 and especially on comparison of the $\chi^{2}$ values relative to the various fitting options in Tables 1 and 2. For all galaxies the fit by a pure $R^{1 / 4}$ law is the worst, with significant residuals. For one galaxy (NGC 4458) the $R^{1 / 4}+$ exponential model gives a marginally better fit with respect to the $R^{1 / n}$ model $\left(\chi^{2}=0.74\right.$ vs. $\left.\chi^{2}=0.85\right)$, but for this galaxy also the pure $R^{1 / 4}$ law and the $f_{\infty}$ models give reasonable fits ( $\chi^{2}=1.13$ and 0.71 , respectively); not surprisingly, this 

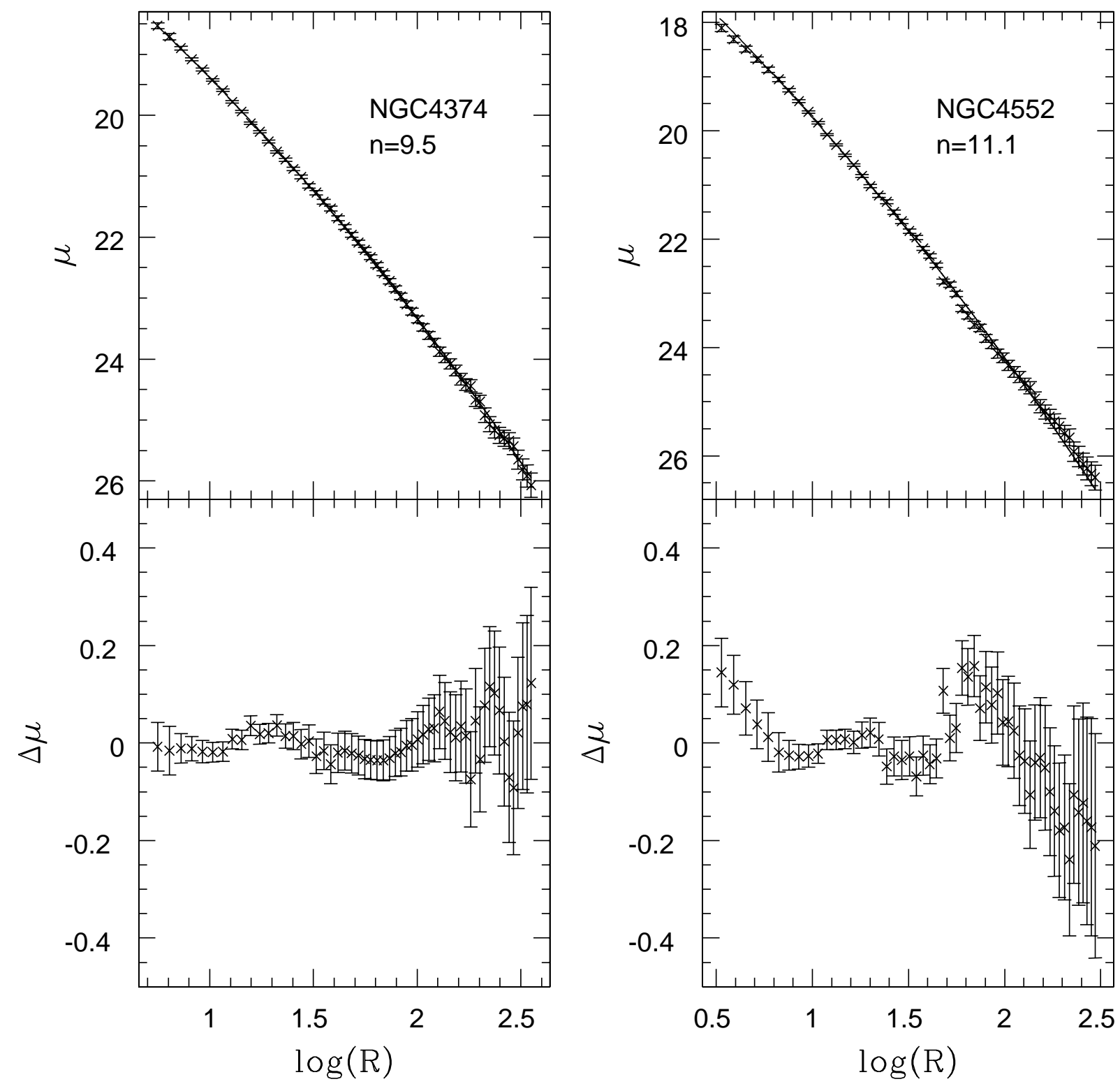

Fig. 2. $R^{1 / n}$ fit. $R$ is in arcsec, $\mu$ in $\operatorname{mag} / \operatorname{arcsec}^{2}$.

Table 1. $R^{1 / n}$ best fits. Circularized profiles, extended radial range, no $L$ constraint. $R_{\mathrm{e}}$ is expressed in arcsec.

\begin{tabular}{ccccccccc}
\hline \hline NGC & $\chi^{2}$ & $R_{\mathrm{e}}$ & $\phi_{\mathrm{FP}}^{\mathrm{a}}$ & $n$ & $\langle\Delta \mu\rangle$ & $(\Delta \mu)_{\max }$ & $\mu_{\max }-\mu_{\min }$ & $M_{B}^{\mathrm{b}}$ \\
\hline 1379 & 0.31 & 25.7 & -5.33 & 2.38 & 0.042 & 0.086 & 7.64 & -19.51 \\
4374 & 0.46 & 259.0 & -5.08 & 9.48 & 0.044 & 0.123 & 7.54 & -21.35 \\
4458 & 0.85 & 22.2 & -5.57 & 3.19 & 0.044 & 0.115 & 4.76 & -18.45 \\
4552 & 1.32 & 104.0 & -5.13 & 11.14 & 0.095 & 0.239 & 8.30 & -20.73 \\
\hline
\end{tabular}

${ }^{\text {a }} \phi_{\mathrm{FP}}$ is given by Eq. (6), with $R_{\mathrm{e}}$ in $\operatorname{arcsec}$ and $S B_{\mathrm{e}}$ in $\mathrm{mag} / \operatorname{arcsec}^{2}$.

b Absolute magnitude in the Blue band (apparent magnitude and extinction from de Vaucouleurs et al. (1991), adopted distance of $18.3 \mathrm{Mpc}$ ).

is the galaxy with the smallest surface brightness excursion $\left(\mu_{\max }-\mu_{\min }\right)$ sampled by the available data (less than $5 \mathrm{mag}$ ) and the one for which the best-fit value of $n(n \approx 3.2)$ is closest to the standard value of 4 . For the three remaining galaxies (NGC 1379, NGC 4374,
NGC 4552), for which the surface brightness profiles extend over a range of more than $7 \mathrm{mag}$, the $R^{1 / n}$ model definitely gives the best fit, even if it has fewer parameters than the $R^{1 / 4}+$ exponential model. In addition, the two objects (NGC 4374 and NGC 4552) that turn out to 
Table 2. Comparison of best-fit models. Circularized profiles, extended radial range, no $L$ constraint. Columns from second to fourth refer to $R^{1 / 4}$ models, from fifth to ninth to $R^{1 / 4}+$ exponential, and last four to $f_{\infty}$ models. $R_{\mathrm{e}}$ is expressed in arcsec.

\begin{tabular}{cccc|ccccc|cccc}
\hline \hline NGC & $\chi^{2}$ & $R_{\mathrm{e}}$ & $\phi_{\mathrm{FP}}$ & $\chi^{2}$ & $R_{\mathrm{e}}^{\mathrm{a}}$ & $\phi_{\mathrm{FP}}$ & $L_{\exp } / L_{\text {tot }}$ & $R_{\text {exp }} / R_{1 / 4}$ & $\chi^{2}$ & $R_{\mathrm{e}}$ & $\phi_{\mathrm{FP}}$ & $\Psi$ \\
\hline 1379 & 5.85 & 34.9 & -5.33 & 0.78 & 27.9 & -5.31 & 0.22 & 0.65 & 3.22 & 32.6 & -5.30 & 9.6 \\
& & & & & & & & & $(5.22)$ & $(46.9)$ & $(-5.38)$ & $(5.2)$ \\
4374 & 9.05 & 75.2 & -4.96 & 0.80 & 104.0 & -4.99 & 0.06 & 0.06 & 1.56 & 98.5 & -4.94 & 7.3 \\
4458 & 1.13 & 26.6 & -5.57 & 0.74 & 21.4 & -5.55 & 0.13 & 0.96 & 0.71 & 23.9 & -5.53 & 9 \\
4552 & 12.23 & 41.2 & -5.07 & 3.07 & 58.1 & -5.10 & 0.10 & 0.09 & 2.86 & 146.4 & -5.22 & 6.3 \\
\hline
\end{tabular}

a Effective radius of the combined profile.

Table 3. Best-fit $R^{1 / n}$ models under various fitting options. $R_{\mathrm{e}}$ is expressed in arcsec.

\begin{tabular}{cccc|cccc|cccc}
\hline \hline NGC & $n^{\mathrm{a}}$ & $R_{\mathrm{e}}$ & $\phi_{\mathrm{FP}}$ & $\chi^{2}$ & $n^{\mathrm{b}}$ & $R_{\mathrm{e}}$ & $\phi_{\mathrm{FP}}$ & $\chi^{2}$ & $n^{\mathrm{c}}$ & $R_{\mathrm{e}}$ & $\phi_{\mathrm{FP}}$ \\
\hline \hline 1379 & 2.88 & 26.8 & -5.32 & 0.38 & 2.36 & 25.4 & -5.33 & 0.31 & 2.39 & 25.7 & -5.33 \\
4374 & 5.73 & 124.1 & -5.00 & 0.33 & $14.5^{\mathrm{d}}$ & 1092 & -5.25 & 0.45 & 9.31 & 247.0 & -5.07 \\
4458 & 4.61 & 29.8 & -5.57 & 0.83 & 3.28 & 22.7 & -5.57 & 0.81 & 3.21 & 22.3 & -5.57 \\
4552 & 5.87 & 56.7 & -5.08 & 0.85 & 9.95 & 95.2 & -5.12 & 1.33 & 11.9 & 119.9 & -5.15 \\
\hline
\end{tabular}

a Extended radial range, curves of growth, no $L$ constraint (constant photometric error assumed, see Appendix B.1).

b Reduced radial range $\left(\mu_{\max }-\mu_{\min }=4.5 \mathrm{mag}\right)$, circularized profile, no $L$ constraint.

c Extended radial range, constrained luminosity $L$, circularized profile.

d Solution "crossing" (see description in Appendix B.3).

be characterized by high values of $n$ (of order 10), when fitted by the $R^{1 / 4}+$ exponential model, would lead to a disk component with an unusually high central brightness $\left(18.2\right.$ and $18.1 \mathrm{mag} / \operatorname{arcsec}^{2}$ in the Blue band, respectively), at variance with the central brightness of galaxy disks that characterize spiral galaxies. It would be interesting to check wheter this is a common feature of $R^{1 / 4}$ + exponential decompositions of early-type galaxies.

\subsection{Robustness of a photometric indicator}

In the discussion of the properties of the FP, it has often been noted (see Kelson et al. 2000a; Treu et al. 2001a; see also Trujillo et al. 2001) that much of our uncertainties in relation to the parameters derived by fitting the photometric profile is resolved by an unexpected circumstance, that is the empirical robustness of the photometric indicator that enters the FP with respect to the modeling procedure. The relevant indicator is

$\phi_{\mathrm{FP}}=\log R_{\mathrm{e}}-\beta S B_{\mathrm{e}}$,

with $\beta \approx 0.32$ (see Eq. (1) and following remarks).

An important point should be noted here. There are two different ways of considering possible variations of $\phi_{\mathrm{FP}}$ for a given object, on the basis of the definition of $S B_{\mathrm{e}}$ reported just before Eq. (1). One is that of considering both $R_{\mathrm{e}}$ and $L$ as model quantities, both determined by the fit to the data (see the comments on the extreme case of NGC 4374 made at the end of the previous subsection). The other is to keep the total luminosity as fixed (for the set of four galaxies studied earlier in this paper, see the last column of Table 1), and let only $R_{\mathrm{e}}$ be determined by the fit. In this latter case, it is readily shown that the change in the value of the photometric indicator associated with a small change of $R_{\mathrm{e}}$ due to the modeling procedure is $\delta \phi_{\mathrm{FP}}=(1-5 \beta) \log e \delta R_{\mathrm{e}} / R_{\mathrm{e}} \approx-0.26 \delta R_{\mathrm{e}} / R_{\mathrm{e}}$; this shows that the change in $\phi_{\mathrm{FP}}$ is, in this case, systematically smaller than the relative change in $R_{\mathrm{e}}$. No matter which definition of $\delta \phi_{\mathrm{FP}}$ we refer to, the impact of a variation in $\phi_{\mathrm{FP}}$ on the FP itself can be quantified by stating that, if we call $\mathcal{R}$ the ratio between the value of $R_{\mathrm{e}}$ predicted by the $\mathrm{FP}$, at a given value of $\sigma_{0}$, and the observed value, then $\delta \mathcal{R} / \mathcal{R}=-\delta \phi_{\mathrm{FP}} / \log e \approx-2.3 \delta \phi_{\mathrm{FP}}$.

Here, based on the first definition of $S B_{\mathrm{e}}$ (for which $L$ is taken as a model quantity), we confirm the general validity of the statement. Surprisingly, the photometric indicator is robust both with respect to changes of the adopted photometric model (compare Table 1 and Table 2) and with respect to variations in the fitting procedure within the same photometric model (compare Table 1 and Table 3). Therefore, for the purpose of measuring the quantities entering the FP we are on safe grounds, whereas for the purpose of interpreting the FP we are left in a rather ambiguous situation.

\subsection{The kinematical counterpart. Low-luminosity galaxies as a physically separate class of objects}

The investigation provided earlier in this paper sheds some light on the issues of modeling and confirms that homology cannot be considered a strict rule for earlytype galaxies. However, the discussion so far has been restricted to the photometric aspects, while the possibility of weak homology (i.e., of systematic trends in structural and 

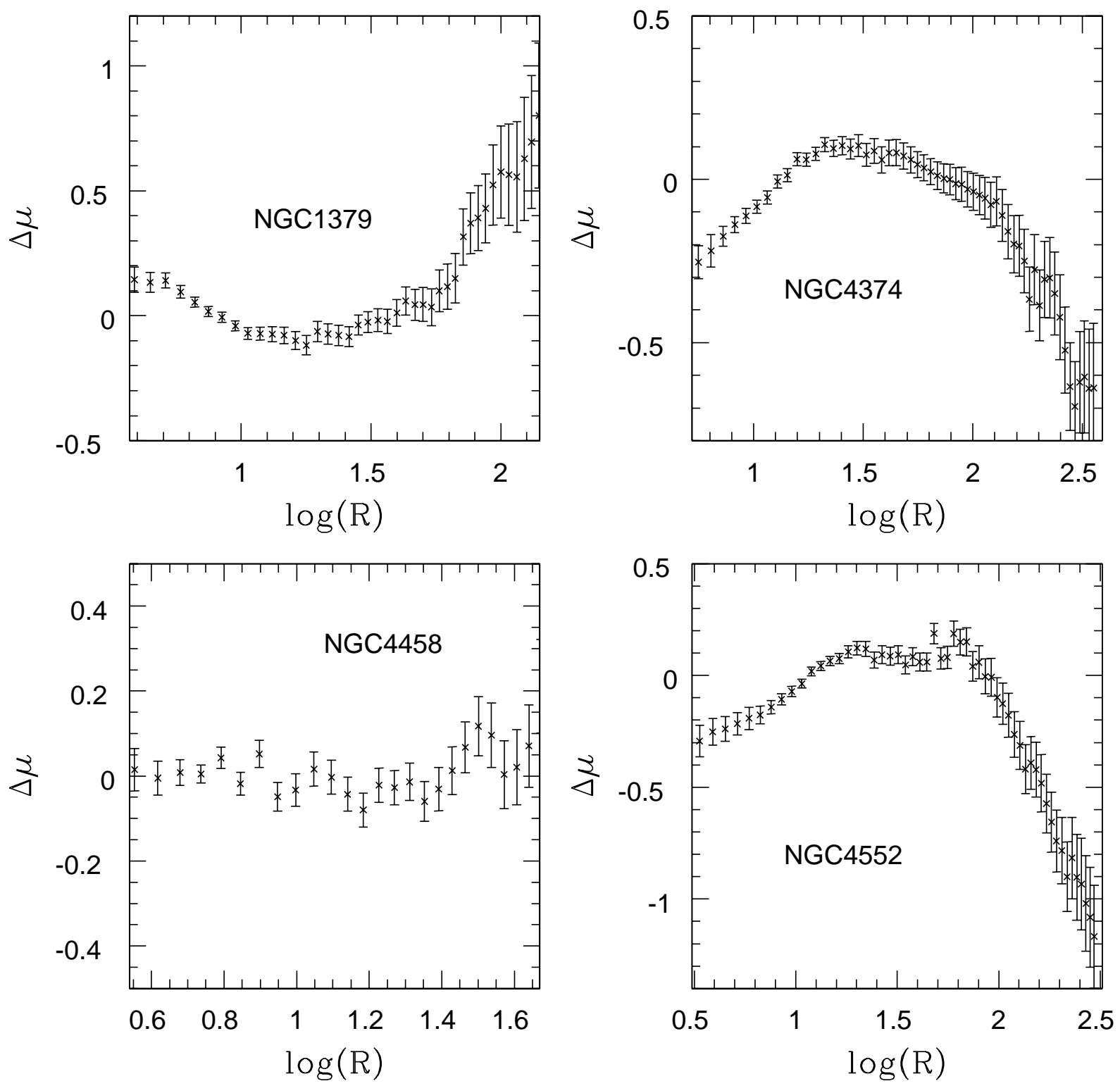

Fig. 3. $R^{1 / 4}$ fit. $R$ is in arcsec, $\mu$ in mag/ $\operatorname{arcsec}^{2}$.

dynamical properties as a function, e.g., of galaxy luminosity) should be assessed by considering the entire set of quantities that enter the FP. In particular, it is unavoidable that one should address the internal kinematical properties of the galaxies involved. In fact, it has long been known that low-luminosity ellipticals are likely to be rotation supported systems (Davies et al. 1983). If we are interested in drawing a connection between the low values of $n$ that best describe low-luminosity ellipticals and the dynamical coefficient that enters the virial theorem, we should not forget that the virial theorem relation reflects the entire dynamical structure of the system involved.

To be more specific, it is often argued that the relevant dynamical coefficient can be conveniently calculated under the simplifying assumption that the system be characterized by an $R^{1 / n}$ profile in projection, while intrinsically the system be spherical, non-rotating, and strictly isotropic. From the Jeans equations such coefficient is determined in a straightforward manner (see PS97; Ciotti \& Lanzoni 1997; see also Sect. 3 and Appendix D below). In practice, it remains quite doubtful that such coefficient can be usefully inserted into a realistic discussion of the FP, given the fact that the velocity dispersion profiles thus calculated are often qualitatively different from those observed (see Appendix C). Indeed, some of the difficulties that we may find in placing the four galaxies described in this section into the context of the FP, as will be attempted in Sect. 3 (in particular, see Figs. 6-9), may just be due to this basic modeling problem.

So far we may claim that we have a reasonable representation of the dynamical structure only of bright ellipticals, at least in the case of intrinsically fairly round objects (e.g., see Bertin \& Stiavelli 1993). Low-luminosity ellipticals appear to be a physically separate class of objects. Until we gain, by systematic spectro-photometric surveys and by new and accurate modeling, a good 

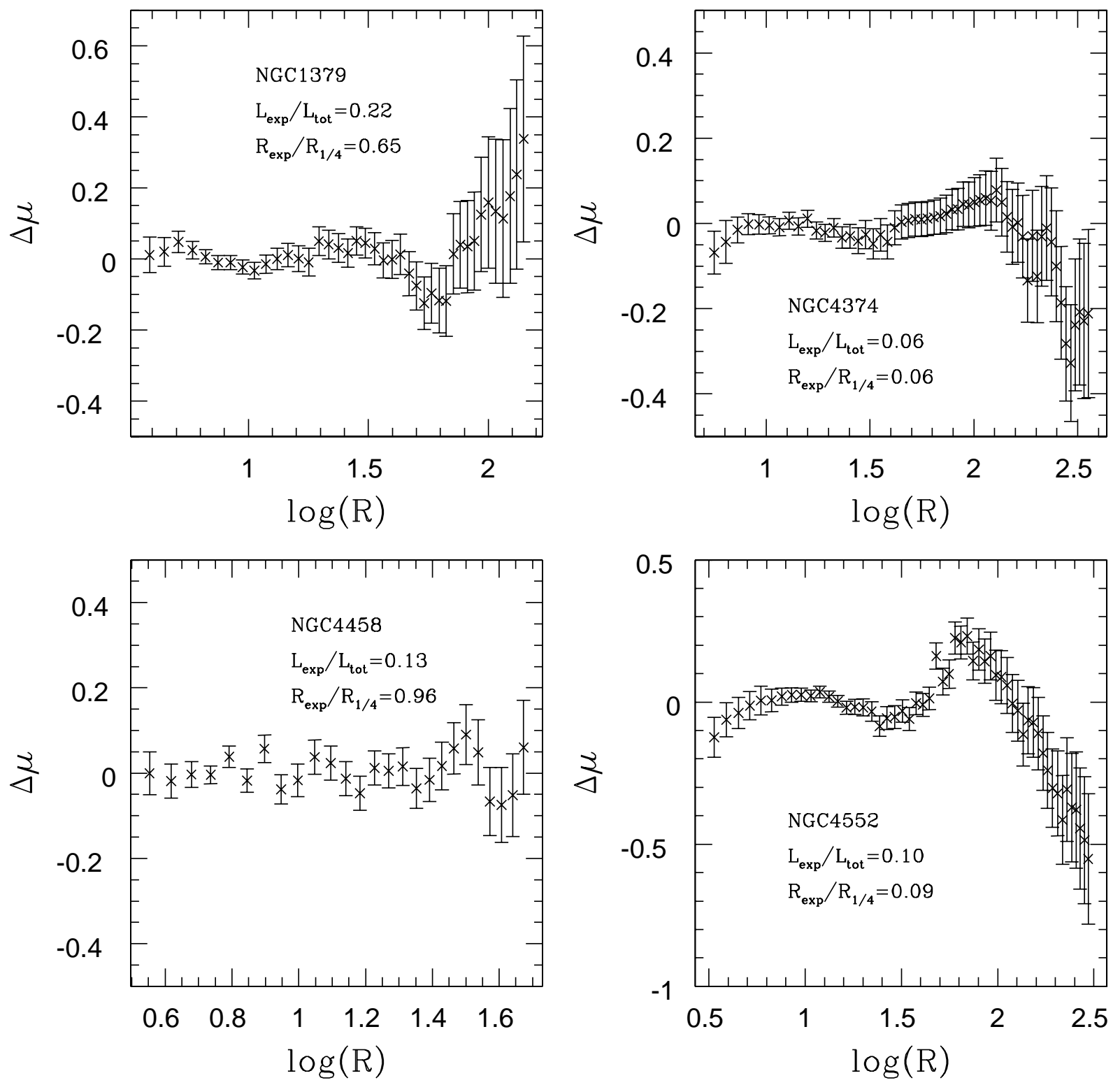

Fig. 4. $R^{1 / 4}+$ exponential fit. $R$ is in arcsec, $\mu$ in mag/arcsec ${ }^{2}$.

picture of how early-type galaxies are structured, from faint to bright objects, there is no hope that we can make a reliable discussion of the impact of the observed deviations from the $R^{1 / 4}$ law on the FP. Nonetheless, in the absence of such comprehensive dynamical modeling, by using the one-component, spherical, non-rotating, isotropic $R^{1 / n}$ models (even if we know them to be an inadequate representation) we will be able to demonstrate, in the next section, that the absence of strict $R^{1 / 4}$ homology can be consistent with an entire spectrum of possible interpretations of the FP. Curiously, these interpretations may well hold even if the empirical relation $n=n(L)$ is affected by large scatter.

\section{Impact on the Fundamental Plane}

In order to assess the consequences of weak homology on the interpretation of the FP, below we follow a line of thought already introduced, e.g., by Ciotti \& Pellegrini (1992).

For a stationary stellar system, the scalar virial theorem can be written as

$\frac{G \Upsilon_{*} L}{R_{\mathrm{e}}}=K_{\mathrm{V}} \sigma_{0}^{2}$

where the coefficient $K_{\mathrm{V}}$ takes into account projection effects, the specific star orbit distribution and the specific density distribution, and effects related to the presence of dark matter; here $\Upsilon_{*}$ is the stellar mass-to-light ratio in the given band used for the determination of $L$ and $R_{\mathrm{e}}$. Equations (1) and (7) imply that in real galaxies, no matter how complex their structure is, the dimensionless factor $\Upsilon_{*} / K_{\mathrm{V}}$ is a well-defined function of any two of the 

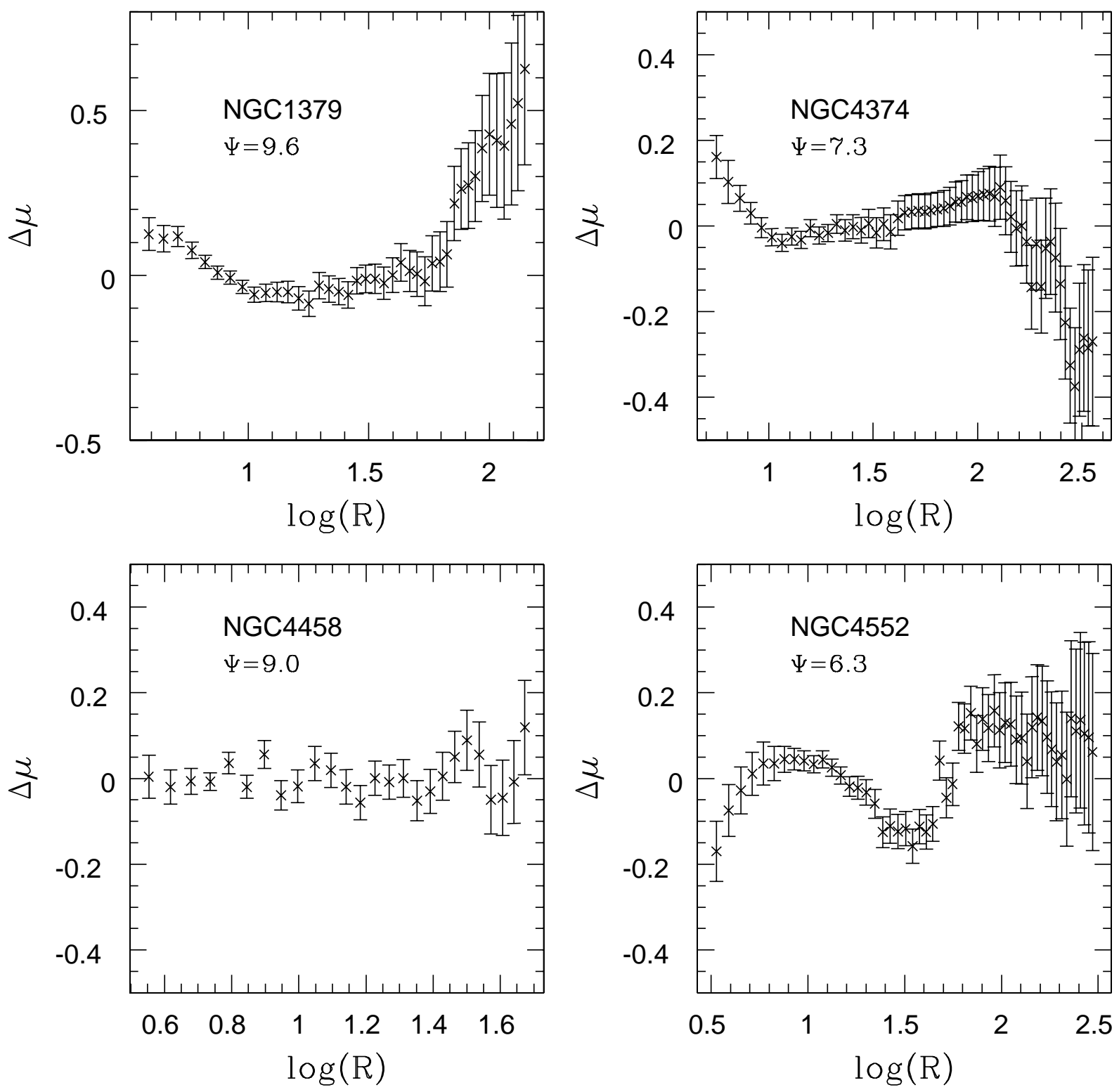

Fig. 5. $f_{\infty}$ fit. $R$ is in arcsec, $\mu$ in mag/arcsec ${ }^{2}$.

three observables $\left(L, R_{\mathrm{e}}, \sigma_{0}\right)$. For example, by eliminating $\sigma_{0}$ from Eqs. (1) and (7) we obtain

$\frac{\Upsilon_{*}}{K_{\mathrm{V}}} \propto R_{\mathrm{e}}^{(2-10 \beta+\alpha) / \alpha} L^{(5 \beta-\alpha) / \alpha}$.

This fact is remarkable by itself. All stellar systems described by Eq. (7) are in virial equilibrium, but only those stellar systems for which $\Upsilon_{*} / K_{\mathrm{V}}$ scales according to Eq. (8) are placed onto the observational manifold given by Eq. (1). Of course, this requirement is only necessary for a model to describe real galaxies. In fact, the FP is not uniformly populated by galaxies; instead, observed stellar systems populate only a well-defined region of the FP.

After recalling two simple extreme interpretations of the FP (Sect. 3.1), in Sect. 3.2 we move to a more general exploration, which will bring out once more a problem of fine tuning met in the study of galaxies.

\subsection{Two extreme interpretations of the Fundamental Plane}

The possibility of simple extreme explanations of the FP is associated with the fact that the exponent of the effective radius appearing in Eq. (8) is observationally very small, i.e., $(2-10 \beta+\alpha) / \alpha \approx 0.04$ in the Blue band.

A first possibility to reproduce the scaling relation in Eq. (8) is then to assume homology (i.e., $K_{\mathrm{V}}$ identical for all galaxies), a variable stellar mass-to-light ratio (see, e.g., Renzini \& Ciotti 1993; van Albada et al. 1995), and the empirically suggested identity $2-10 \beta+\alpha=0$. With these assumptions we find

$\Upsilon_{*} \propto L^{\delta}$

here $\delta=(2-\alpha) / 2 \alpha \simeq 0.30 \pm 0.064$, where the scatter has been calculated by standard error propagation techniques. From a physical point of view, in a simple (coeval 
and single metallicity) monolithic formation, passive evolution scenario, a mass-luminosity relation for galaxies, of the kind suggested by Eq. (9), can result if, with significant fine tuning, the basic properties of the Initial Mass Function, which describes the origin of stellar populations, properly correlate with galaxy mass.

An alternative extreme explanation can be proposed by assuming a constant stellar mass-to-light ratio $\Upsilon_{*}$ and the existence of weak homology (see Ciotti \& Pellegrini 1992; CCD93; Renzini \& Ciotti 1993; Djorgovski 1995; Hjorth \& Madsen 1995; Ciotti et al. 1996; PS97; and references therein). In this case it is the quantity $K_{\mathrm{V}}$ (instead of $\Upsilon_{*}$ ) that is required to be a well-defined function of $R_{\mathrm{e}}$ and $L$. If we take $2-10 \beta+\alpha=0$, the required dependence is

$K_{\mathrm{V}} \propto L^{-\delta}$,

with $\delta$ and its scatter the same as above. As noted in the Introduction, this latter interpretation is qualitatively encouraged by the observations. Quantitatively, we can easily show that we are in the right ballpark. In fact, from Eq. (10) it is realized that a factor of 20 in $L$, would require a change in $K_{\mathrm{V}}$ by a factor of $\approx 2.45$. If we consider onecomponent, spherical, non-rotating, isotropic $R^{1 / n}$ models and a simulated spectroscopic aperture of $R_{\mathrm{e}} / 8$, the relation $K_{\mathrm{V}}(n)$ can be computed (Ciotti \& Lanzoni 1997; PS97; see Appendix D). An accurate and convenient analytical representation in the range $1 \leq n \leq 10$ (with typical errors on the order of one percent) is given by

$K_{\mathrm{V}}(n) \simeq \frac{73.32}{10.465+(n-0.94)^{2}}+0.954$.

It is then demonstrated that the observed excursion in $n$ (see Sect. 2) could well be consistent with the desired range in $K_{\mathrm{V}}$. A similar argument also holds for the $f_{\infty}$ models, for which we have the following simple analytical interpolation formula, accurate to around one percent,

$K_{\mathrm{V}}(\Psi) \simeq \frac{142.3-41.51 \Psi+2.66 \Psi^{2}}{30.61-10.7 \Psi+\Psi^{2}}$

in the range $2 \leq \Psi \leq 10$.

From this simple discussion we might conclude that weak homology of elliptical galaxies might indeed be consistent with a constant $\Upsilon_{*}$ and the FP.

Unfortunately, the determination of the coefficient $K_{\mathrm{V}}$ is sensitive to the choice of models (see the expressions recorded above and Appendix D) and to the choice of the aperture radius. For the $R^{1 / n}$ models, the latter dependence is not too significant, even in the presence of the well-known unrealistic central depression in their projected velocity dispersion profiles (see Appendix C); this has been checked by comparing the values of $K_{\mathrm{V}}$ obtained on the basis of an $R_{\mathrm{e}} / 10$ aperture with those based on $R_{\mathrm{e}} / 8$. In order to make a physically better justified case, one might think it more appropriate to refer to the $f_{\infty}$ models, which are known to possess realistic kinematical profiles. Note that this would lead to significantly different answers with respect to the application of $R^{1 / n}$ models. For example (see Appendix D), if we take NGC 4552, the $R^{1 / n}$ modeling would give $n \simeq 11.14$ and $K_{\mathrm{V}} \simeq 1.6$, while the $f_{\infty}$ modeling would set $\Psi \simeq 6.2$ and $K_{\mathrm{V}} \simeq 2.5$; for the galaxy NGC 4458 (closest to the standard $R^{1 / 4}$ law in our sample), we find $n \simeq 3.2$ and $K_{\mathrm{V}} \simeq 5.7$ based on $R^{1 / n}$ modeling or $\Psi \simeq 9, K_{\mathrm{V}} \simeq 3$ based on $f_{\infty}$ modeling. Here we prefer not to pursue this line of investigation, because the empirical relation $\Psi=\Psi(L)$ has not yet been studied systematically and because, in any case, we know a priori that such modeling, even if it might turn out to be partially successful, would lack physical justification when applied to low-luminosity, rotation-supported galaxies.

\subsection{A more general interpretation allowed by weak homology}

We now describe a more general interpretation of the FP allowed by the existence of weak homology. For simplicity, we refer here to the family of $R^{1 / n}$ models that has led to Eq. (11), although the method proposed could be implemented for any well-defined family of dynamical models. In particular, here we explore the theoretical manifold obtained by mapping, with the aid of Monte Carlo simulations, the model space $\left(n, \Upsilon_{*}, L, R_{\mathrm{e}}\right)$ into the observed space $\left(L, R_{\mathrm{e}}, \sigma_{0}\right)$. The domain of model galaxies considered in the simulations is defined by $1 \lesssim R_{\mathrm{e}} \lesssim 20$, $0.1 \lesssim L \lesssim 50$, and $100 \lesssim \sigma_{0} \lesssim 350$, where radii are measured in kpc, luminosities in $10^{10}$ solar Blue luminosities, and velocities in $\mathrm{km} \mathrm{s}^{-1}$. For each four-dimensional point $\left(n, \Upsilon_{*}, L, R_{\mathrm{e}}\right)$ in model space we calculate $\sigma_{0}$ from Eq. (7) and then we check if the mapped (virialized) system belongs to the FP. This allows us to select in model space, as acceptable candidates for real galaxies, only those points that turn out to be compatible, in the observed space, with the FP correlation and the observed scatter around Eq. (1). We also explore the "backbone" model manifold obtained by artificially reducing the FP thickness to a limiting zero-scatter case. For simplicity, we plot the projections of the manifold thus derived on the natural planes $\left(L, \Upsilon_{*}\right)$ and $(L, n)$, which are precisely the two planes that are used to illustrate the two extreme interpretations described in Sect. 3.1.

We start this exploration by applying the proposed technique to the two cases discussed in the previous subsection. The case of $R^{1 / 4}$ homology is studied by restricting the model population to a set of points for which $3.99 \leq n \leq 4.01$. The derived dependence of $\Upsilon_{*}$ on $L$ is well fitted by the power law relation of Eq. (9), with $\delta \simeq 0.27$ (consistent with the value $0.30 \pm 0.064$ argued in Sect. 3.1), with reduced $\chi^{2} \simeq 1.7$. The scatter of points along the vertical axis at fixed $L$ (see Fig. 6) reflects the assumed thickness of the FP. Indeed, if we artificially reduce the scatter in $R_{\mathrm{e}}$ from 0.15 to 0.01 , the derived power-law exponent becomes $\delta=0.28$, with reduced $\chi^{2} \simeq 7 \times 10^{-2}$ (the thick locus of points in Fig. 6). 


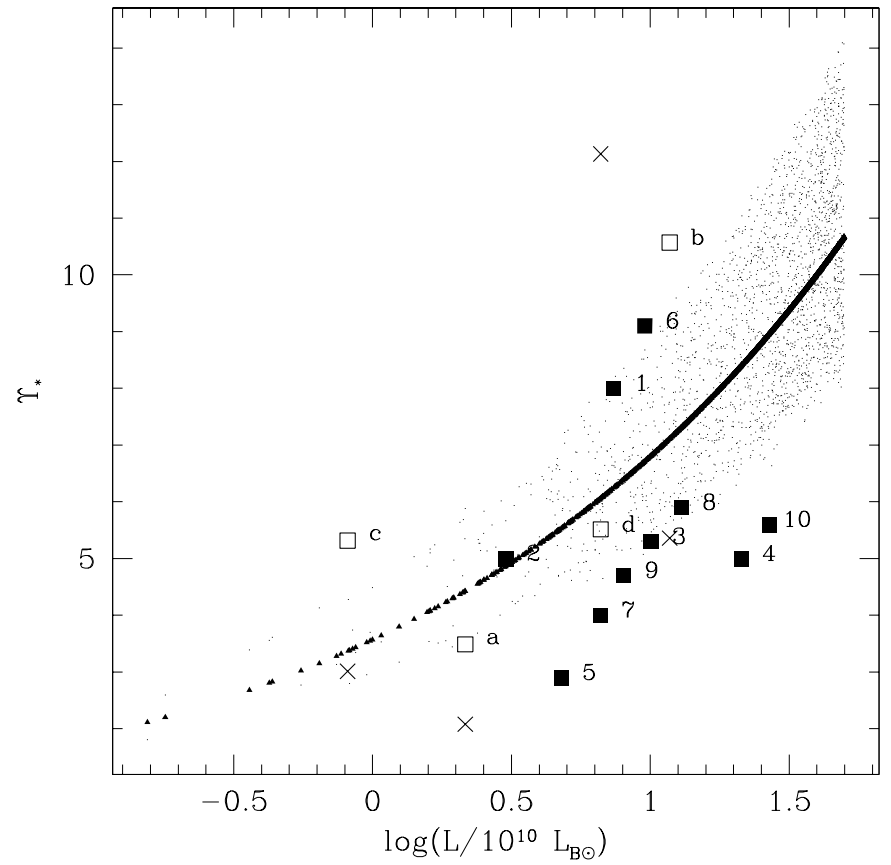

Fig. 6. Projection of the manifold in model space identified by $R^{1 / 4}$ homology. The scatter of points reflects the adopted scatter around the FP in the observed space. The symbols that trace the thick line represent the "backbone" manifold. Open squares represent the four galaxies of Sect. 2, following the $R^{1 / n}$ modeling summarized in Tables 1 and D.1; crosses represent the same four galaxies, following the $f_{\infty}$ modeling summarized in Tables 2 and D1 (see Sect. 3.3 for additional description). Filled squares represent the sample of ten galaxies considered in Table 5 of Bertin et al. (1994); see Sect. 3.4 of the present paper for a description.

To illustrate the second case (see Fig. 7), we pick a constant stellar mass-to-light ratio $3.49 \leq \Upsilon_{*} \leq 3.51$. The selected points are quite dispersed along the $n$ axis. A good fit is obtained by a law of the form $n=a+b \log L$, with $b \simeq 4.02$ and $\chi^{2} \simeq 1.3$, not so well in agreement with the observed scaling relation given in Eq. (3). After artificially reducing the scatter around the FP to 1 percent in $R_{\mathrm{e}}$, we now have $b \simeq 5.03$ and $\chi^{2} \simeq 0.24$. (By increasing or decreasing the reference value of $\Upsilon_{*}$, the simulation points would move below or above the strip identified by the present choice of $\Upsilon_{*}$.)

A somewhat unexpected result of these simple simulations is that the relatively small dispersion of $R_{\mathrm{e}}$ for real galaxies, around the value associated with the FP at any fixed $L$ and $\sigma_{0}$, is consistent with a significantly larger dispersion of the model parameters along the vertical axes in Figs. 6 and 7 at fixed values of $L$. Therefore, weak homology (e.g., a correlation between the structural parameter $n$ and the luminosity $L$ ) can be at the origin of a relatively thin FP, even in the presence of a substantial dispersion of $n$ at given $L$.

At this point we can proceed to consider the most general case, where neither homology is strict nor mass-tolight ratio is constant. In fact, we leave the model points to run over the entire ranges of $\Upsilon_{*}$ values and $n$ values in

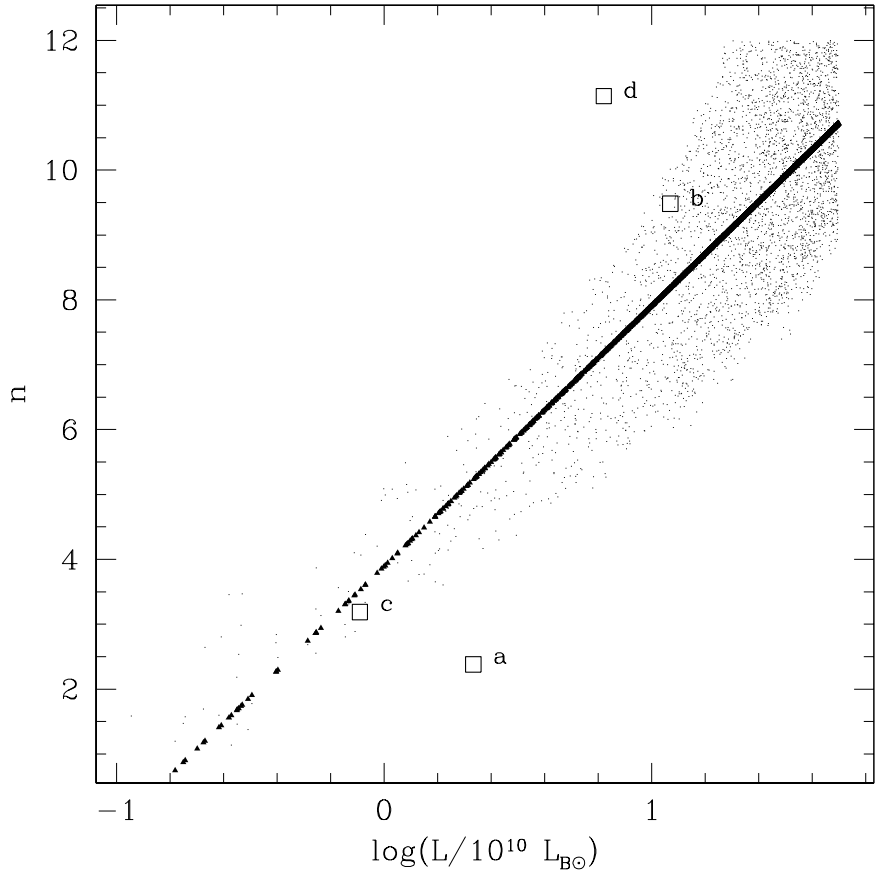

Fig. 7. Projection on the plane $(L, n)$ of the manifold in model space identified by the constant mass-to-light ratio assumption $\Upsilon_{*} \simeq 3.5$. The scatter of points reflects the adopted scatter around the FP in the observed space. Solid symbols represent the "backbone" manifold. Open squares represent the four galaxies of Sect. 2, following the $R^{1 / n}$ modeling summarized in Tables 1 and D1 (see Sect. 3.3 for additional description).

the domain indicated at the beginning of this subsection. For simplicity, we look for the backbone manifold selected by artificially reducing the scatter on the observed $R_{\mathrm{e}}$ to 0.01 . In this way the scatter presented by the points on the two natural planes $\left(L, \Upsilon_{*}\right)$ and $(L, n)$ will be determined only by projection effects (of the relevant manifold onto the desired planes).

In Fig. 8 we show the projection of what we have called the backbone manifold onto the $\left(L, \Upsilon_{*}\right)$ plane. Note how, at any given value of $L$, a larger range of $\Upsilon_{*}$ values is permitted, even if the FP considered in the simulations is extremely thin. Therefore, in the general case, $\Upsilon_{*}$ is not necessarily well correlated with galaxy luminosity, as a result of the presence of the free parameter $n$. It is interesting to check how models with different $n$ are distributed inside this projection of the backbone manifold; the open circles represent models characterized by $3.8 \leq n \leq 4.8$, a range slightly broader than that shown in the homology case shown in Fig. 6. In any case, also under the more general circumstances considered here, the FP relation is associated with an overall increasing trend for the stellar mass-to-light ratio $\Upsilon_{*}$ with $L$.

Similar comments apply to the projection illustrated in Fig. 9. Here the open circles represent models with the stellar mass-to-light ratio $\Upsilon_{*} \approx 3.5$. Note the large spread in $n$ present, still consistent with the existence of a thin FP, once the restriction of nearly constant $\Upsilon_{*}$ is relaxed. Note also that there remains only a mild trend 


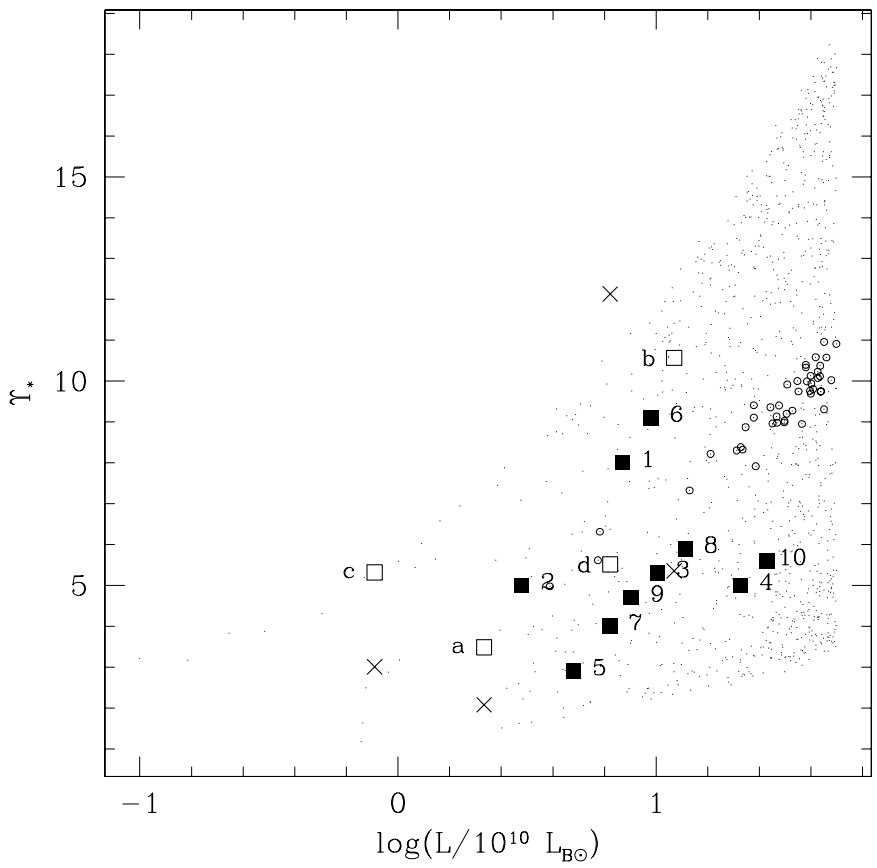

Fig. 8. Projection on the $\left(L, \Upsilon_{*}\right)$ plane of the entire backbone manifold identified by the FP with scatter artificially reduced to 0.01 . Open circles correspond to models with $3.8 \leq n \leq$ 4.2. Open squares, crosses, and filled squares define observed galaxies as in Fig. 6 .

of $n$ with $L$ required by the existence of the FP, but only if we focus on the zero-scatter limit. Since in reality some correlation is observed (see Introduction and Eq. (3)), we should conclude that something else, in addition to the FP, characterizes the underlying weak homology of real galaxies.

\subsection{Locating the four prototypical objects without detailed stellar dynamical modeling}

With the aim of a better appreciation of the naive indications provided by a pure photometric modeling we have recorded, in Figs. 6-9, as open squares (labels $a, b, c, d$ following the order of Table 1), the points available for the set of four galaxies discussed in the previous part of the paper and from the available data about their central velocity dispersions. This information (see Table D.1), based on the $R^{1 / n}$ modeling, allows us to extract values of $\Upsilon_{*}$ from the observed values of $\sigma_{0}(119,296,106$, and $269 \mathrm{~km} \mathrm{~s}^{-1}$ for NGC 1379, 4374, 4458, and 4552, respectively, McElroy 1995). In Figs. 6 and 8 we also record the different points that would be argued on the basis of a one-component $f_{\infty}$ photometric modeling.

For the purposes of this section, the relevant quantities associated with the four galaxies are rescaled, when necessary, to the long cosmological distance scale. This is consistent with the adopted choice of coefficients for the FP (see Eq. (1) and following comments) and with the analysis to be presented in the following subsection.

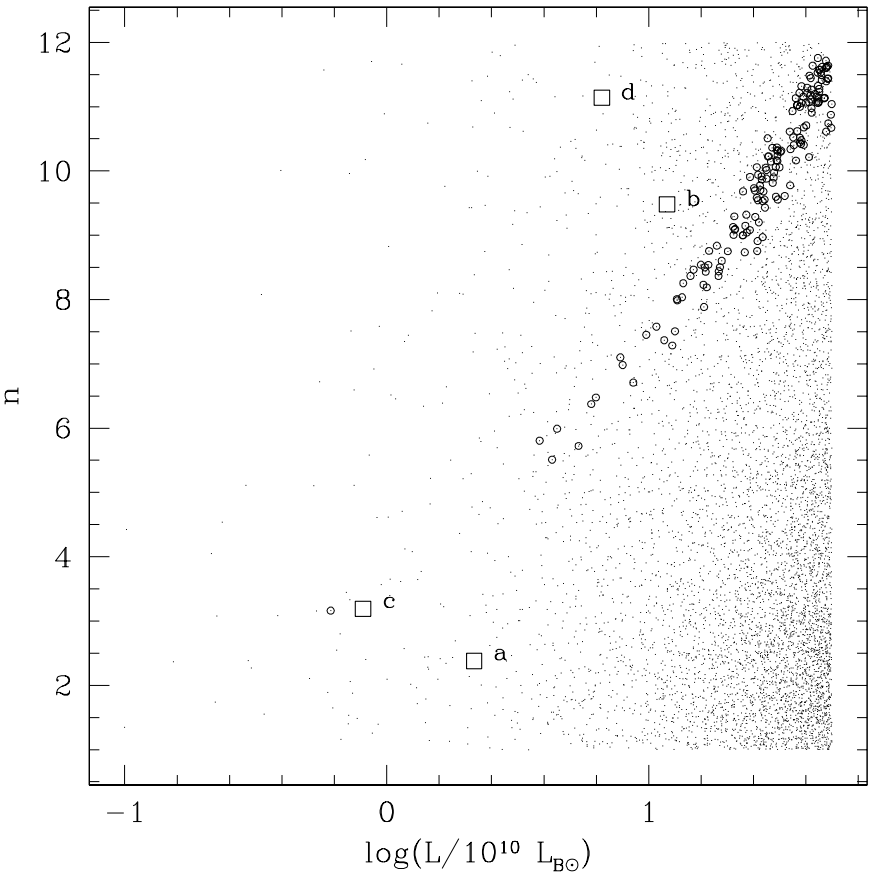

Fig. 9. Projection on the $(L, n)$ plane of the entire backbone manifold identified by the FP with scatter artificially reduced to 0.01 . Open circles correspond to models with $3.4 \leq \Upsilon_{*} \leq$ 3.6. Open squares define observed galaxies as in Fig. 7 .

\subsection{Trends from a sample of relatively bright galaxies and the role of dark matter}

In order to better relate the arguments provided in this Section with the trends observed in real galaxies, we have included in Figs. 6 and 8 some "data-points" that are external to the simulations described above. These datapoints have been obtained as follows.

We have referred back to the results of an ESO Key Programme "A search for dark matter in elliptical galaxies" and, in particular, to a set of 10 galaxies the properties of which are summarized in Table 5 of Bertin et al. (1994). The objects are: NGC 1399, NGC 3379, NGC 4374, NGC 4472, NGC 5812, NGC 5813, NGC 7507, NGC 7626, NGC 7796, and IC 4296 (some of these are also part of the sample considered by Gerhard et al. 2001). The numbers from 1 to 10 of the filled squares in Figs. 6 and 8 represent precisely these objects in the order followed above. These galaxies had been considered as those for which extensive and reliable stellar dynamical modeling was available, on the basis of accurate and radially extended stellar dynamical data. We borrow the values of $\Upsilon_{*}$ and $R_{\mathrm{e}}$ listed in Table 5 of Bertin et al. (1994) under the columns $\left(M_{\mathrm{L}} / L_{\mathrm{B}}\right)_{2 C}$ and $R_{\mathrm{e}}^{m}$, respectively, and thus obtain the values of $K_{\mathrm{V}}$ for those objects, based on a detailed stellar dynamical modeling. One object, NGC 4374, is also part of the set of four galaxies for which we have re-examined the photometric profile in Sect. 2 .

We note that those models did include significant amounts of dark matter. Therefore, it is not surprising to find now that several filled squares fall outside the strip 
outlined in Fig. 6, which has been calculated on the basis of strict $R^{1 / 4}$ homology. Then Fig. 8 shows that the same data-points are well consistent with a possible weak homology that is neither the strict $R^{1 / 4}$ homology nor the one based on a constant $\Upsilon_{*}$. Note that the broad area outlined in Fig. 8 has been obtained by reducing artificially the scatter of the FP to 0.01 .

\section{Conclusions}

This paper, organized in two parts, has led to a number of results, although many are the questions that remain only partly answered. Here we provide a summary of the main points made in the paper and of the most important questions that are left for future investigations.

In the first part of the paper we have re-examined the surface brightness profiles of NGC 1379, NGC 4374, NGC 4458, and NGC 4552, four galaxies that are known to present significant deviations from the $R^{1 / 4}$ law. We have considered the relative performance of four different photometric models, namely the $R^{1 / n}, R^{1 / 4}, R^{1 / 4}+$ exponential, and $f_{\infty}$ models. Each of the four different photometric models has been tested against a variety of options for the fitting procedure. We have thus reached the conclusion that there is indeed convincing evidence that homology cannot be considered a strict rule for early type galaxies. In particular, we have found that:

- For the four galaxies of this limited sample the fit by a pure $R^{1 / 4}$ law is the worst.

- For three objects the $R^{1 / n}$ photometric model definitely provides the best fit, even better than that by the $R^{1 / 4}+$ exponential model, although the latter model has the advantage of one additional free parameter.

- For NGC 4458, which is the object with the smallest surface brightness excursion available (less than five magnitudes), the $R^{1 / 4}+$ exponential model gives a marginally better fit with respect to the $R^{1 / n}$ model; but, for this, the $R^{1 / 4}$ and the $f_{\infty}$ models give practically equivalent fits.

- The $f_{\infty}$ models manage to provide a reasonable description of the bright ellipticals even when the $R^{1 / n}$ modeling suggests a value of $n$ significantly larger than 4. However, they do not appear to be viable for the low-luminosity ellipticals, which are characterized by a small value of $n$. This does not come as a surprise, because the physical arguments that have led to the construction of the $f_{\infty}$ models reflect the picture of low rotation, collisionless collapse, while low-luminosity ellipticals are known to be generally rotation supported.

- The values of the effective radius resulting from the fit can change dramatically (by factors of up to three), depending on the fitting procedure and on the adopted photometric model. On the other hand, we confirm that the determination of the photometric indicator, i.e. the combination of photometric quantities entering the $\mathrm{FP}$, is robust.
The good performance of the $R^{1 / n}$ model in describing the photometric profiles of galaxies deviating from the $R^{1 / 4}$ law and the existence of an empirical correlation between $n$ and $L$ (see Eq. (3), based on a sample of 52 galaxies) suggest that elliptical galaxies might be considered as weakly homologous systems. Of course, a clear indication of weak homology cannot be based on photometric properties alone. In the second part of the paper, we have then addressed the impact of weak homology on the physical interpretation of the FP, starting from a re-discussion of the rationale behind two extreme interpretations of the tilt of the FP. One is obtained by assuming strict homology and a suitable relation between the stellar mass-to-light ratio and the galaxy luminosity. The other is obtained by taking a constant stellar mass-to-light ratio (from galaxy to galaxy) and a suitable form of weak homology. The latter interpretation usually raises concerns (besides the problems related to the interpretation of the observed correlations involving luminosity, such as the color-luminosity relation; see discussion in the Introduction), because the large scatter around the correlation $n=n(L)$ appears to be hard to reconcile with the small dispersion observed around the FP. We have thus obtained the following results:

- In order to address the problem in its generality, we have developed a systematic approach to the study of the FP, based on a mapping between model and observed quantities. For simplicity, we have used, as a guiding tool for the present investigation, simple one-component, spherically symmetric, isotropic $R^{1 / n}$ models. The study would be best carried out with other families of models, better justified from the physical point of view, but for the present purposes the simple $R^{1 / n}$ models used provide an adequate demonstration. The use of one-component models is equivalent to assuming that dark matter, if present, is distributed in direct proportion to the luminous matter.

- Within this framework, we have shown how the scatter of the stellar mass-to-light ratio (in the first of the two simplest interpretations) or of the index $n$ (in the second interpretation) is fully controlled by the observed thickness of the FP. This quantifies in detail the problem of parameter fine tuning that has to be faced.

- We have then relaxed the hypothesis of a simple (i.e., one-parameter) origin of the tilt of the FP and focused, for simplicity, on the structure of the FP with artificially reduced thickness. A fine tuning problem obviously remains, because the existence of the FP imposes a strict condition on $\Upsilon_{*} / K_{\mathrm{V}}$. However, we have been able to demonstrate that the ranges of variation for the stellar mass-to-light ratio and the index $n$ of the models, at fixed galaxy luminosity, can be substantial and still be consistent with an arbitrarily thin FP. The fine tuning involved requires that variations in the stellar mass-to-light ratio be compensated for by corresponding variations in galaxy structure. 
- Preliminary indications provided by a set of "data points" associated with a sample of 14 galaxies suggest that neither the strict homology nor the constant stellar mass-to-light solution are a satisfactory explanation of the observed Fundamental Plane.

This paper opens the way to the following new investigations, to be carried out in separate papers:

- A study based on the approach introduced in Sect. 3 within a more complex dynamical structure (presence of dark matter and more complicated geometries for the model galaxies).

- A study of the evolution of the FP with redshift, within a physical interpretation based on weak homology. If the observations were able to tell the time evolution of the coefficients $\alpha$ and $\beta$ in the exponents in Eq. (8), then, from our mapping procedure, we could infer the actual evolution shared between luminosity and structural parameters. From a different, predictive point of view, if we argue that the tilt of the FP is totally due to weak homology (with constant $\Upsilon_{*}$ from galaxy to galaxy, so that the FP is generated by systematic structural variations with luminosity of the quantity $K_{\mathrm{V}}$ ), then we would expect only a parallel shift of the FP, i.e. only a systematic shift of its zero-point $\gamma$ with time. Clearly, this picture must be only approximately viable, because galaxies follow the metallicity - velocity dispersion relation (see Bender et al. 1993 and references therein) and the color-magnitude relation (see Bower et al. 1992), consistent with the fact that the tilt of the FP depends on waveband (e.g., see the results based on near-infrared data obtained by Pahre et al. 1998b). On the other hand, if, as is frequently done, we refer to the picture where all galaxies are strictly homologous systems, so that the tilt of the FP is completely due to a luminosity dependence of $\Upsilon_{*}$ (at fixed metallicity), then the luminosity evolution of faint galaxies should be faster than that of bright galaxies, and the tilt of the FP would be expected to change with $z$, with the larger changes at the FP faint end. Here several different physical scenarios would be available (see also Renzini \& Ciotti 1993).

- A study of the relation between weak homology and the popular paradigm of galaxy merging.

From the observational point of view, it is especially urgent to carry out a discussion of weak homology, extended to the spectroscopic (stellar-dynamical) data, for a complete sample of nearby early-type galaxies.

Acknowledgements. We would like to thank T. van Albada, R. de Carvalho, M. Lombardi, and T. Treu for very useful comments and suggestions. This work has been partially supported by MURST of Italy (co-fin 2000).

\section{Appendix A: Photometric models}

\section{A.1. $R^{1 / n}$ and $R^{1 / 4}$ models}

Consider the surface brightness profile (Sersic 1968) given by

$$
I(R)=I_{0} \mathrm{e}^{-b \eta^{1 / n}}
$$

where $\eta=R / R_{\mathrm{e}}, n$ is a positive real number, and $b=b(n)$ a dimensionless constant such that $R_{\mathrm{e}}$ is the effective radius, i.e., the projected radius encircling half of the total luminosity associated with $I(R)$. As proved in a separate paper (Ciotti \& Bertin 1999), the first terms of the asymptotic expansion of $b(n)$, for large $n$, are $b(n) \sim 2 n-1 / 3+4 /(405 n)$, already accurate to a part in one thousand for $n$ as low as unity. The proper normalization of the $R^{1 / n}$ profile, to be considered for a case with given scales $L$ and $R^{1 / n}$, is

$$
I(R)=\frac{L}{R_{\mathrm{e}}^{2}} \frac{b^{2 n}}{2 \pi n \Gamma(2 n)} \mathrm{e}^{-b \eta^{1 / n}}
$$

where $\Gamma$ represents the complete Gamma function. The photometric profile is thus characterized by two scales (e.g., $L$ and $R_{\mathrm{e}}$ ) and one dimensionless parameter $(n)$. The standard de Vaucouleurs (1948) profile is obtained by setting $n=4$.

\section{A.2. Two-component $R^{1 / 4}+$ exponential models}

For the surface brightness profile we take here the superposition of an $R^{1 / 4}$ component and a standard "exponential disk", i.e.

$$
\begin{aligned}
I(R)= & I_{1 / 4}(0) \exp \left[-b(4)\left(R / R_{1 / 4}\right)^{1 / 4}\right] \\
& +I_{\exp }(0) \exp \left[-b(1)\left(R / R_{\exp }\right)\right],
\end{aligned}
$$

where $R_{1 / 4}$ and $R_{\exp }$ are the effective radii of the $R^{1 / 4}$ and of the exponential component, respectively. By fitting this model to an observed photometric profile, we can derive the total luminosities $L_{\exp }, L_{1 / 4}$ associated with the separate components and from these the total luminosity $L_{\text {tot }}=L_{1 / 4}+L_{\text {exp }}$. In conclusion, this two-component photometric model is characterized by two scales $\left(L_{\text {tot }}\right.$ and $R_{\mathrm{e}}$; for the latter quantity we refer to the effective radius for the combined profile) and by two dimensionless parameters (e.g., $L_{\mathrm{exp}} / L_{\text {tot }}$ and $R_{\exp } / R_{1 / 4}$ ).

\section{A.3. $f_{\infty}$ models}

The $f_{\infty}$ models are dynamical models applicable to collisionless stellar systems. They have been constructed (Bertin \& Stiavelli 1984) by following the physical scenario that elliptical galaxies may have formed through collisionless collapse (van Albada 1982). In the spherical limit, their anisotropic distribution function is given by

$f_{\infty}= \begin{cases}A(-E)^{3 / 2} \exp \left(-a E-c J^{2} / 2\right) & \text { if } E \leq 0 \\ 0 & \text { if } E>0\end{cases}$ 
where $E=v^{2} / 2+\Phi(r)$ and $J$ are the star energy and the magnitude of the star angular momentum, per unit mass, respectively. Two of the three available dimensional constants $(A, a$, and $c)$ set the physical scales of the model (e.g., the total mass and the half-mass radius $r_{\mathrm{M}}$ ). The remaining dimensionless parameter, for example written as $\gamma=a c /(4 \pi G A)$, then characterizes the one-parameter family of $f_{\infty}$ models. The solution of the Poisson equation provides the relation $\gamma=\gamma(\Psi)$, where $\Psi$ denotes the dimensionless central potential $\Psi=-a \Phi(0)$. In practice, under the assumption that the stellar mass-to-light ratio is constant within the galaxy and after projection for comparison with the observations, the relevant photometric profile associated with the $f_{\infty}$ models, in addition to a luminosity scale $(L)$ and a length scale $\left(R_{\mathrm{e}} \approx r_{\mathrm{M}} / 1.3\right)$, depends on one dimensionless parameter only $(\Psi)$. A detailed discussion of the properties of this equilibrium sequence is given by Bertin \& Stiavelli (1993). Here we limit our discussion to $f_{\infty}$ models characterized by $2 \leq \Psi \leq 10$. In fact, models with $\Psi<2$ are unstable, while, on the other hand, models with $\Psi \geq 10$ are very similar to each other on the large scale, except for the development of a nuclear concentration on scales much smaller than those studied in this paper.

Given the focus of the present paper on the $R^{1 / n}$ photometric models, as a preliminary investigation we have compared the photometric profiles of the $f_{\infty}$ dynamical models (treated as "the galaxies") to those of the $R^{1 / n}$ models (taken as the "fitted models"). We have thus obtained the best-fit $n(\Psi)$ by minimizing the quantity

$\chi^{2}=\sum_{\eta_{\min }}^{\eta_{\max }}\left[\mu_{\infty}(\eta)-\mu_{1 / n}(\eta)\right]^{2}$,

where $\eta=R / R_{\mathrm{e}}$ is expressed in units of the effective radius of the $f_{\infty}$ models. The adopted radial interval is $0.1 \leq \eta \leq 10$; note that the adopted outer radius is well far out with respect to the radius $R_{\max }$ typically considered for observed photometric profiles.

We found that for $f_{\infty}$ models with $\Psi \gtrsim 5$ a fit over the adopted radial interval can be obtained with $(\Delta \mu)_{\max }<$ 0.4 . In contrast, for $\Psi \lesssim 5$, in order to obtain a fit with $(\Delta \mu)_{\max }<0.4$ we had to reduce the radial interval to typical values $0.23 \leq \eta \leq 4$. This smaller radial range is actually applicable to realistic conditions. In these latter cases of low- $\Psi$ models we found that the best-fit value of $n$ is sensitive to the specific radial interval adopted; for example, the quality condition $(\Delta \mu)_{\max }<0.4$ can be satisfied for a different radial range (say $0.1 \leq \eta \leq 2$ ), but the resulting best-fit value of $n$ turns out to be significantly lower. In Fig. A.1 we show the optimal fitting relation $n=n(\Psi)$ for the fits based on the full radial range, for $\Psi \geq 5.5$, and on the reduced radial range $(0.23 \leq \eta \leq 4)$ for $\Psi<5.5$. Note how close to the de Vaucouleurs profiles, $n=4$, are the high- $\Psi$ models, as realized from the very beginning (see Bertin \& Stiavelli 1993).

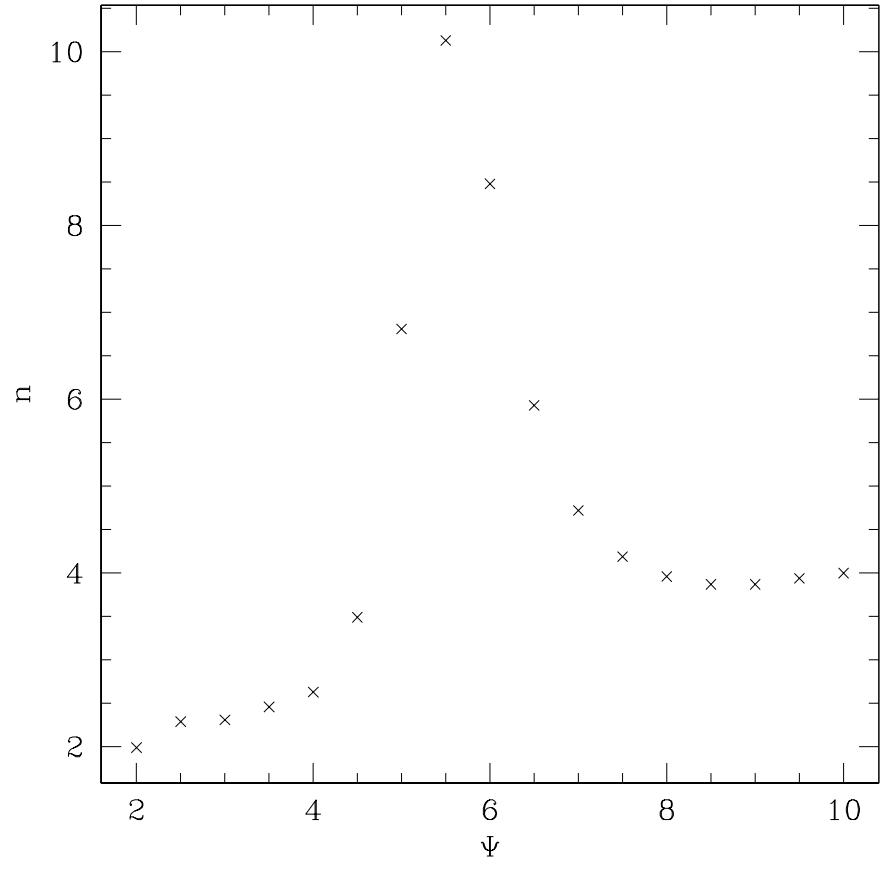

Fig. A.1. The best-fit $n$, obtained by fitting the $f_{\infty}$ models, projected along the line of sight, with $R^{1 / n}$ profiles, as a function of the model dimensionless central potential $\Psi$. The radial interval adopted in the fit is described in the text. Note the "plateau" at $n=4$ reached by high- $\Psi$ models.

\section{Appendix B: Fitting procedures}

In this appendix we briefly describe different options to fit a model, chosen within the set of models presented in Appendix A, to the photometry of observed objects available within a given spatial range.

\section{B.1. Profiles, circularized profiles, curves of growth}

In the procedure of profile fitting, we generally refer to circularized profiles. The objects selected for the present study are fairly round in projection. Therefore, the differences between the photometric profiles taken along the short (with coordinate $R_{\mathrm{s}}$ ) or the long (with coordinate $R_{1}$ ) axis (as preferred by CCD93, DCC94) with respect to the profiles obtained by referring to the circularized radius $R=\sqrt{R_{\mathrm{s}} R_{\mathrm{l}}}$ are rather small.

The observed profiles in mag $/ \operatorname{arcsec}^{2}, \mu_{\text {obs }}(R)=$ $-2.5 \log I_{\mathrm{obs}}(R)$, are compared to the corresponding model profiles $I_{\text {mod }}$

$\mu_{\text {mod }}(R)=-2.5 \log I_{\text {mod }}(R)$.

Following standard methods (e.g., see Bertin et al. 1988), the best-fit parameters are determined by minimizing the $\chi^{2}$ function defined as:

$\chi^{2}=\sum_{i=1}^{N} \frac{\left[\mu_{\mathrm{obs}}\left(R_{i}\right)-\mu_{\mathrm{mod}}\left(R_{i}\right)\right]^{2}}{\sigma_{\mathrm{obs}}^{2}\left(R_{i}\right)\left(N-N_{\mathrm{par}}\right)}$

where $N$ is the number of data-points in the interval $\left(R_{\min }, R_{\max }\right), \sigma_{\text {obs }}\left(R_{i}\right)$ represents the photometric error 
on $\mu_{\mathrm{obs}}\left(R_{i}\right)$, and the factor $\left(N-N_{\text {par }}\right)$ takes into account the number of free parameters in the fit. The formal errors $\delta_{i}$ associated with the best-fit values of the parameters $x_{i}$ involved can be calculated from the covariance matrix $E=2 H^{-1}$, where $H$ is defined as

$H_{i j}=\left(N-N_{\text {par }}\right) \frac{\partial^{2} \chi^{2}}{\partial x_{i} \partial x_{j}}$.

Therefore, we have

$\delta_{i}=\sqrt{E_{i i}}$,

while the off-diagonal terms provide the correlation coefficients.

The parameters thus determined (e.g., the effective radius $R_{\mathrm{e}}$ ) do not represent the quantities implied by their ideal definitions (e.g., the projected radius of the disk that encloses half of the total luminosity), because the fit is performed only on a limited radial range $\left(R_{\min }, R_{\max }\right)$. In this procedure the model parameters (e.g., $n, R_{\mathrm{e}}$, and $I_{0}$ for the $R^{1 / n}$ models) are taken to be independent of each other.

A second fitting procedure (preferred, e.g., by PS97) refers to the so-called curve of growth, i.e. to the function associated with the integrated luminosity $L(R)=$ $2 \pi \int_{0}^{R} R^{\prime} I\left(R^{\prime}\right) \mathrm{d} R^{\prime}$. One then compares the data points for $m_{\mathrm{obs}}(R)=-2.5 \log L_{\mathrm{obs}}(R)$, expressed in magnitudes, to the corresponding curve of growth $m_{\bmod }(R)$ derived from the photometric model. It is clear that this procedure involves a major difference with respect to the profile fitting procedure, because the data points of the curves of growth cannot be treated as independent of each other. If we proceed naively, but improperly, by means of a $\chi^{2}$ function defined as in Eq. (B.2), (but in the results shown in Sect. 2.3 we took a constant photometric error) with $\mu$ replaced by $m$, we find "best-fit values" of the relevant parameters that can be significantly discrepant with respect to those found from profile fitting (compare the values of $n$ given in the second column of Table 3 with those recorded in Table 1). The discrepancies follow the general trends reported in the literature (see Introduction and PS97).

\section{B.2. Constraints}

One of the tests that we have performed on our set of observed photometries has been the study of the dependence of the best-fit parameters on the radial range of the fit. In order to compare with earlier studies (e.g., see Burkert 1993), we have thus considered a case where all photometric profiles for the four galaxies studied in this paper were limited to a common excursion of $4.5 \mathrm{mag}$ (see second group of columns in Table 3).

Another natural possibility (see Bertin et al. 1988) is to consider a fit under the constraint that the observed profile and the model profile be characterized by the same integrated luminosity within the radial range of the fit. To these fits we refer as fits with constrained luminosity (see
Sect. 2, third group of columns in Table 3). Explicitly, we impose:

$\int_{R_{\min }}^{R_{\max }} R I_{\mathrm{obs}}(R) \mathrm{d} R=\int_{R_{\min }}^{R_{\max }} R I_{\bmod }(R) \mathrm{d} R$

so that the luminosity scale is set as a constraint and the number of free parameters with respect to which the $\chi^{2}$ function is minimized is reduced by one.

Similarly, for the fit to the curves of growth it is natural to impose that $L_{\text {obs }}\left(R_{\max }\right)=L_{\bmod }\left(R_{\max }\right)$.

\section{B.3. Comments}

Not unexpectedly, when free parameters are available, such as $n$ or $\Psi$, the search for the best-fit model actually leads to several possible minima of the relevant $\chi^{2}$ function. In Table 2 we have recorded the properties (in parentheses) of a secondary minimum available for NGC 1379, within the $f_{\infty}$ models, which appears to be particularly significant. In Tables 1 and 3 we have considered only the $R^{1 / n}$ models corresponding to the absolute minima found. For each galaxy, we have also checked the properties of other (typically 4 or 5) models corresponding to secondary minima, with $\chi^{2}$ within a factor of $3-4$ with respect to that associated with the absolute minimum. The related model parameters turn out to be significantly different only when the associated value of $\chi^{2}$ changes significantly. The values of $n$ and $R_{\mathrm{e}}$ thus identified tend to increase, or decrease, together, but the photometric parameter $\phi_{\mathrm{FP}}$ remains robustly stable.

The case illustrated briefly in the second group of columns in Table 3, that of the reduced radial range, presents, not surprisingly, the most complex behavior. Here we can even witness the occurrence of "crossing" of solutions. An example of this phenomenon is provided by the galaxy NGC 4374 . For this object the absolute minimum over the reduced radial range is obtained for $n \approx 14.5$ and $R_{\mathrm{e}} \approx 1092$, with $\chi^{2} \approx 0.34$ (see Table 3 ). A very near (when considering the $\chi^{2}$ value) secondary minimum is $n \approx 9.9$ and $R_{\mathrm{e}} \approx 302$, with $\chi^{2} \approx 0.55$. When fitting the profile of the same galaxy over the extended radial range the absolute minimum is found (see Table 1 ) at $n \approx 9.5$ with $R_{\mathrm{e}} \approx 259$ and $\chi^{2} \approx 0.46$. Thus, the solution corresponding to the absolute minimum obtained over the the whole available radial range can turn out to be associated with a secondary minimum when the fit is extended over a reduced radial range.

\section{Appendix C: General properties of the velocity dispersion profiles of simple dynamical models}

If we wish to draw consequences, from the observed trends in the photometric profiles, on the structure of the FP, we have to take into account some dynamical information. In practice, for the objects under consideration we have to estimate the relevant virial coefficient (see Appendix D). Since the dynamical quantity that appears in the FP is the 
central (projected) velocity dispersion (based on a suitable small aperture), it is clear that our consequences will depend mostly on the assumed behavior, for the velocity dispersion profiles, in the vicinity of the galactic center. In this Appendix we will show that if we start from a given density profile and then proceed to infer, as commonly done (through the Jeans equations under simple assumptions on the pressure tensor; e.g., see PS97), the velocity dispersion profile, the resulting kinematical profile is very sensitive to the specific limiting behavior (at very small radii) of the density profile we consider. In many cases the implied kinematical profile may be completely unrealistic (e.g., by developing a major "central hole", not observed; see Bailey \& MacDonald 1981). This sensitivity is unfortunate, because it makes it necessary to address in detail the dynamical basis of the photometric models used to fit the observations. In this respect, we should recall here that the velocity dispersion profiles of the $f_{\infty}$ models (see Appendix A.3) are (generally realistic) monotonic, decreasing with radius.

In the following we focus on spherically symmetric, isotropic, one-component galaxy models with finite mass and, for simplicity, limit our discussion to the unprojected velocity dispersion only.

Suppose that the model density profile at small radii is characterized by the asymptotic behavior

$\rho(r) \sim r^{-\alpha}$

with $0 \leq \alpha<3$. From the explicit solution of the appropriate hydrostatic equilibrium condition,

$$
\frac{\mathrm{d}}{\mathrm{d} r}\left[\rho(r) \sigma_{r}^{2}(r)\right]=-G \frac{\rho(r) M(r)}{r^{2}},
$$

it is readily shown that for $\alpha=0$ the velocity dispersion reaches a finite (non-vanishing) value at the center (dependent on the shape of the entire density profile). For $0<\alpha<1$ we find

$\sigma_{r}^{2}(r) \sim r^{\alpha}$

and so $\sigma_{r}^{2}(0)=0$ and a "central hole" is present (note that all $R^{1 / n}$ models fall in this case, because close to the center their density profile is characterized by $\alpha=1-1 / n$ ). When $\alpha=1$, we have

$\sigma_{r}^{2}(r) \sim-r \ln (r)$

and so the central value of the velocity dispersion vanishes. Finally, for $1<\alpha<3$ the relation is

$\sigma_{r}^{2}(r) \sim r^{2-\alpha}$

Thus, for $1<\alpha<2$ the central velocity dispersion vanishes and a hole occurs; when $\alpha=2$ the central velocity dispersion is finite (its value depends on the shape of the entire density profile, as in the case $\alpha=0$ ), and for $2<\alpha<3$ it diverges.
In order to obtain an estimate of the velocity dispersion gradient close to the center, we can rewrite Eq. (C2) as

$\frac{\mathrm{d} \sigma_{r}^{2}}{\mathrm{~d} r}=-G \frac{M(r)}{r^{2}}-G \frac{1}{\rho^{2}} \frac{\mathrm{d} \rho}{\mathrm{d} r} \int_{r}^{\infty} \frac{\rho(x) M(x)}{x^{2}} \mathrm{~d} x$.

Let us now focus on the case $\alpha=0$, and thus express the density profile as $\rho(r) \sim \rho(0)-\rho_{1} r^{\beta}$. Then, it can be shown that for $0<\beta<2$ there is indeed a hole in the velocity dispersion; in turn, for $\beta=2$ the direction of the slope of the velocity dispersion depends on the density profile of the entire model, while for $\beta>2$ the velocity dispersion is monotonically decreasing with radius.

We may add that, independently of the slope of the stellar density profile in the central regions, if a massive black hole is present, then the slope of the square of the velocity dispersion is -1 , and so, at least in the central regions, no hole is present in the velocity dispersion profile.

By experimenting with more general density profiles in the central regions, we have also encountered the possibility of "wavy" velocity dispersion profiles.

\section{Appendix D: The virial coefficient $K_{V}$}

After this short kinematical digression, it should be clear that the virial coefficient $K_{\mathrm{V}}=G M /\left(R_{\mathrm{e}} \sigma_{0}^{2}\right)$ that controls the relation between the virial theorem and the FP is sensitive to the dynamical model that we adopt to describe galaxies, not just to the photometric signature as captured, for example, by the $R^{1 / n}$ profiles. In addition, within a given set of dynamical models, the virial coefficient can be sensitive to the precise definition that we adopt for "central velocity dispersion".

To set the basis for our simulations of Sect. 3, we have computed the virial coefficient for the isotropic $R^{1 / n}$ models, with $\sigma_{0}$ based on an aperture of radius $R_{\mathrm{e}} / 10$. In addition, to illustrate the above-mentioned sensitivity problem we have computed the same coefficient also for the $f_{\infty}$ models. We have checked that, for the isotropic $R^{1 / n}$ models, the numbers are in agreement with those reported earlier by PS97. The results are summarized in Fig. D.1.

From this figure, an immediate warning in relation to the sensitivity problem derives from inspection of the high$\Psi$ models: while with respect to the photometric performance the models with $7 \leq \Psi \leq 10$ are all very well fitted by $R^{1 / 4}$ profiles (see Fig. A.1), their virial coefficient is found to vary significantly, from $\approx 2.3$ to $\approx 3.3$.

Another simple way to point out quantitatively the embarassing modeling problem that we are facing is given in Table D.1. Here we record, for the four galaxies studied in this paper, the value of the virial coefficient obtained by referring to the best-fit $R^{1 / n}$ models suggested by Table 1, to those of Table 3 (b: reduced radial range), or to the best-fit $f_{\infty}$ models identified in Table 2 . Note that if we modeled these galaxies as isotropic $R^{1 / 4}$ models, they would all be assigned the same virial coefficient $K_{1 / 4} \approx 4.6$. We should emphasize that, in the spirit of the studies of the FP, all these inferences are made without 


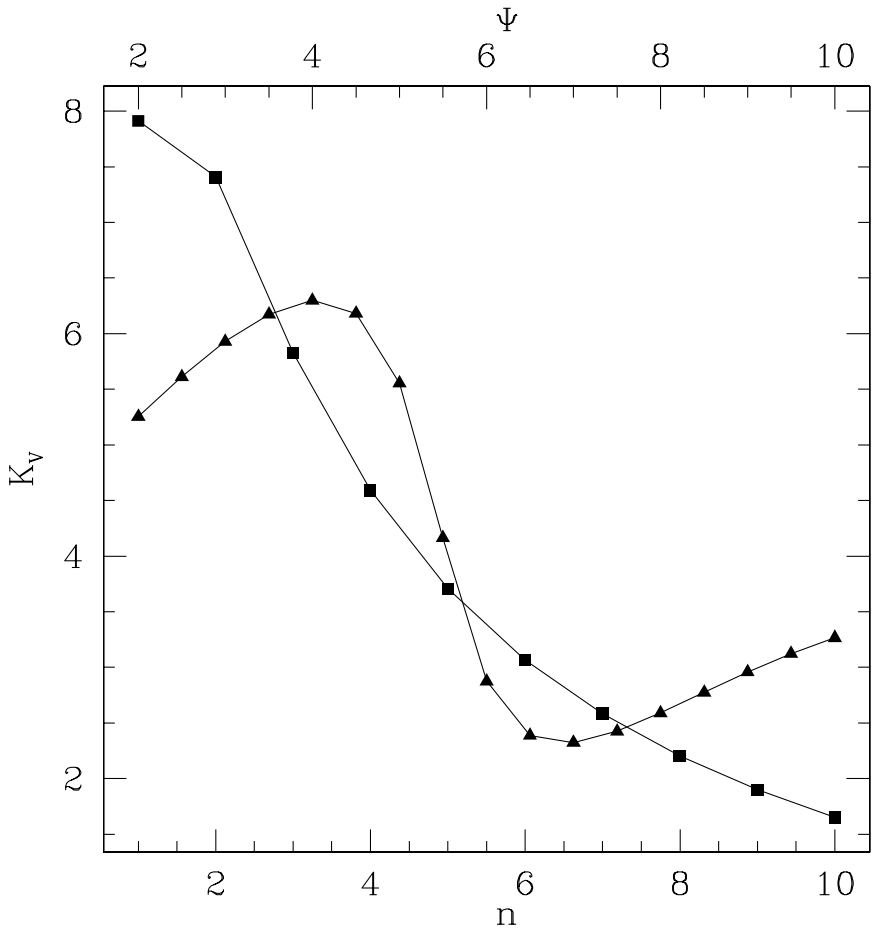

Fig. D.1. The virial coefficient for the $f_{\infty}$ (triangles) and the isotropic $R^{1 / n}$ (squares) models based on an aperture of radius $R_{\mathrm{e}} / 8$.

Table D.1. Virial coefficient $K_{\mathrm{V}}$ corresponding to different fitting procedures.

\begin{tabular}{cccc}
\hline \hline NGC & $\left(R^{1 / n}\right)^{\mathrm{a}}$ & $\left(R^{1 / n}\right)^{\mathrm{b}}$ & $f_{\infty}$ \\
\hline 1379 & 6.8 & 6.8 & 3.2 \\
& & & $(5.1)$ \\
4374 & 1.8 & 1.8 & 2.4 \\
4458 & 5.7 & 5.5 & 3.0 \\
4552 & 1.6 & 1.8 & 2.5 \\
\hline
\end{tabular}

a Extended radial range, no $L$ constraint. See Table 1.

b Reduced radial range, no $L$ constraint. See Table 3.

considering the detailed behavior of the actual velocity dispersion profiles for the galaxies under investigation.

\section{References}

Andredakis, Y. C. 1998, MNRAS, 295, 725

Andredakis, Y. C., Peletier, R. F., \& Balcells, M. 1995, MNRAS, 275, 874

Bailey, M. E., \& MacDonald, J. 1981, MNRAS, 194, 195

Bender, R., Burstein, D., \& Faber, S. M. 1992, ApJ, 399, 462

Bender, R., Burstein, D., \& Faber, S. M. 1993, ApJ, 411, 153

Bender, R., Saglia, R. P., Ziegler, B., et al. 1998, ApJ, 493, 529

Bertin, G., Bertola, F., Buson, L. M., et al. 1994, A\&A, 292, 381

Bertin, G., Saglia, R. P., \& Stiavelli, M. 1988, ApJ, 330, 78

Bertin, G., \& Stiavelli, M. 1984, A\&A, 137, 26

Bertin, G., \& Stiavelli, M. 1993, Rep. Prog. Phys., 56, 493

Bower, R. G., Lucey, J. R., \& Ellis, R. S. 1992, MNRAS, 254, 601
Burkert, A. 1993, A\&A, 278, 23

Caon, N., Capaccioli, M., \& D'Onofrio, M. 1993, MNRAS, 265, 1013 (CCD93)

Caon, N., Capaccioli, M., \& D’Onofrio, M. 1994, A\&A, 106, S199

Caon, N., Capaccioli, M., \& Rampazzo, R. 1990, A\&A, 86, S429

Capaccioli, M. 1987, in Structure and dynamics of elliptical galaxies, ed. P. T. de Zeeuw (Reidel, Dordrecht), 47

Capaccioli, M. 1989, in The world of galaxies, ed. H. G. Corwin, \& L. Bottinelli (Springer-Verlag, Berlin), 208

Capelato, H. V., de Carvalho, R. R., \& Carlberg, R. G. 1995, ApJ, 451, 525

Ciotti, L. 1991, A\&A, 249, 99

Ciotti, L., \& Bertin, G. 1999, A\&A, 352, 447

Ciotti, L., \& Lanzoni, B. 1997, A\&A, 321, 724

Ciotti, L., Lanzoni, B., \& Renzini, A. 1996, MNRAS, 282, 1

Ciotti, L., \& Pellegrini, S. 1992, MNRAS, 255, 561

Courteau, S., de Jong, R. S., \& Broeils, A. H. 1996, ApJ, 457, L73

Davies, I. G., Phillips, S., Cawson, M. G. M., et al. 1988, MNRAS, 232, 239

Davies, R. L., Efstathiou, G., Fall, S. M., et al. 1983, ApJ, 266, 41

de Carvalho, R. R., \& da Costa, L. N. 1988, ApJ, 68, S173

de Vaucouleurs, G. 1948, Ann. d'Astroph., 11, 247

de Vaucouleurs, G., \& Capaccioli, M. 1979, ApJ, 40, S699

de Vaucouleurs, G., de Vaucouleurs, A., Corwin, H. G., et al. 1991, Third Reference Catalogue of Bright Galaxies (Springer-Verlag, New York)

Djorgovski, S. 1995, ApJ, 438, L29

Djorgovski, S., \& Davis, M. 1987, ApJ, 313, 59

D’Onofrio, M., Capaccioli, M., \& Caon, N. 1994, MNRAS, 271, 523 (DCC94)

Dressler, A., Lynden-Bell, D., Burstein D., et al. 1987, ApJ, 313,42

Faber, S. M., Dressler, A., Davies, R. L., et al. 1987, in Nearly normal galaxies, ed. S. M. Faber (Springer, New York), 175

Gerbal, D., Lima-Neto, G. B., Marquez, I., \& Veraghen, H. 1997, MNRAS, 285, L41

Gerhard, O., Kronawitter, A., Saglia, R. P., \& Bender, R. 2001, AJ, 121, 1936

Graham, A. W. 1998, MNRAS, 295, 933

Graham, A. W., Lauer, T. R., Colless, M., \& Postman, M. 1996, ApJ, 465, 534

Graham, A. W., Colless, M., Busarello, G., Zaggia, S., \& Longo, G. 1998, A\&AS, 133, 325

Graham, A. W., \& Colless, M. 1997, MNRAS, 287, 221

Hjorth, J., \& Madsen, J. 1995, ApJ, 445, 55

Jørgensen, I., Franx, M., \& Kjærgaard, P. 1993, ApJ, 411, 34

Jørgensen, I., Franx, M., Hjorth, J., et al. 1999, MNRAS, 308, 833

Kelson, D. D., van Dokkum, P., Franx, M., et al. 1997, ApJ, 478, L13

Kelson, D. D., Illingworth, G. D., van Dokkum, P., et al. 2000a, ApJ, 531, 137

Kelson, D. D., Illingworth, G. D., van Dokkum, P., et al. 2000b, ApJ, 531, 159

Kelson, D. D., Illingworth, G. D., van Dokkum, P., et al. 2000c, ApJ, 531, 184

Khosroshahi, H. G., Wadadekar, Y., Kembhavi, A., \& Mobasher, B. 2000, ApJ, 531, L103

McElroy, D. B. 1995, ApJS, 100, 105 
Makino, J., Akiyama, K., \& Sugimoto, D. 1990, PASJ, 42, 205

Michard, R. 1985, A\&A, 59, S205

Pahre, M. A., \& Djorgovski, S. G. 1997, in The Nature of Elliptical Galaxies, ed. M. Arnaboldi, G. S. Da Costa, \& P. Saha, ASP Conf. Ser., 116, 154

Pahre, M. A., de Carvalho, R. R., \& Djorgovski, S. G. 1998a, AJ, 116, 1606

Pahre, M. A., Djorgovski, S. G., \& de Carvalho, R. R. 1998b, AJ, 116, 1591

Prugniel, P., \& Simien, F. 1997, A\&A, 321, 111 (PS97)

Renzini, A., \& Ciotti, L. 1993, ApJ, 416, L49

Saglia, R. P., Bertschinger, E., Baggley, G., et al. 1997, ApJ, 109, S79

Schombert, J. M. 1986, ApJ, 60, S603

Scorza, C., \& Bender, R. 1995, A\&A, 293, 20

Sersic, J. L. 1968, Atlas de galaxias australes, Observatorio Astronomico, Cordoba

Stiavelli, M., Miller, B. W., Ferguson, H. C., et al. 2001, AJ, 121,1385
Treu, T., Stiavelli, M., Casertano, S., et al. 1999, MNRAS, 308, 1037

Treu, T., Stiavelli, M., Møller, P., et al. 2001a, MNRAS, 326, 221

Treu, T., Stiavelli, M., Bertin, G., et al. 2001b, MNRAS, 326, 237

Trujillo, I., Graham, A. W., \& Caon, N. 2001, MNRAS, 326, 869

van Albada, T. S. 1982, MNRAS, 201, 939

van Albada, T. S., Bertin, G., \& Stiavelli, M. 1995, MNRAS, 276,1255

van Dokkum, P., \& Franx, M. 1996, MNRAS, 281, 985

van Dokkum, P., Franx, M., Kelson, D. D., et al. 1998a, ApJ, 500,714

van Dokkum, P., Franx, M., Kelson, D. D., et al. 1998b, ApJ, 504, L17

Wadadekar, Y., Robbason, B., \& Kembhavi, A. 1999, AJ, 117, 1219

Young, C. K., \& Currie, M. J. 1994, MNRAS, 268, L11 